\author{
Hebe González \\ (Consejo Nacional de Investigaciones \\ Cientificas y Técnicas (CONICET) \\ Universidad Nacional de San Juan (UNSJ))
}

\title{
Una aproximación a la fonología del tapiete (Tupí-Guaraní)
}

\begin{abstract}
This article presents the main features of Tapiete phonology, a Tupi-Guarani language spoken in Argentina, Bolivia and Paraguay. It discusses the segmental phonology focusing on phonemes, their phonetic description, the phonotactics, the rules that govern the allophonic variations and the syllable structure. At the supra-segmental level, the article provides a characterization of nasal harmony and stress.

KEYWORDS: Tapiete; Tupi-Guarani; Phonology; Nasal harmony; Reduplication.
\end{abstract}

\section{RESUMEN}

Este artículo presenta los principales rasgos de la fonología tapiete, lengua tupí-guaraní hablada en Argentina, Bolivia y Paraguay. El artículo aborda la fonología segmental, centrándose en los fonemas, su caracterización fonética, la fonotáctica, las reglas que rigen las variaciones alofónicas y la estructura silábica. A nivel suprasegmental, el artículo brinda una caracterización de la armonía nasal y el acento. Se describen las variaciones morfofonológicas, específicamente, en su relación con la armonía nasal y el acento.

PALABRAS CLAVES: Tapiete; Tupí-Guaraní; Fonología; Armonía nasal; Reduplicación. 


\section{INTRODUCCIÓN}

El tapiete es una lengua tupí-guaraní hablada en Argentina, Bolivia y Paraguay. En la Argentina, el tapiete se habla en la provincia de Salta, particularmente en la ciudad de Tartagal y en la localidad de La Curvita, a orillas del río Pilcomayo, cerca del límite con Bolivia $^{1}$. En la Argentina, la lengua ha sufrido un marcado retroceso de uso en los últimos treinta años. Múltiples factores explican esta situación, entre los que se destaca el alto índice de matrimonios interétnicos en los que, independientemente de que el padre o la madre sean hablantes tapietes, se ha cortado la transmisión intergeneracional de la lengua ${ }^{2}$. Así, en la actualidad, los niños y la mayoría de los adolescentes, sólo poseen un conocimiento pasivo de la lengua, es decir, la entienden pero no la hablan. ${ }^{3}$

Según Aryon Rodrigues (1984/85), el tapiete, junto con el guaraní y el avá-guaraní (también conocido como chiriguano), pertenece al grupo 1 de los 8 que el autor postula al interior de la familia tupí-guaraní. Dietrich (1986), por su parte, considera el tapiete un dialecto del avá-guaraní (chiriguano). Si bien, como asegura Dietrich, el tapiete se encuentra estrechamente emparentado al avá-guaraní (chiriguano), González (2005) propone un desarrollo independiente basado en rasgos fonológicos y morfológicos.

Este artículo presenta los principales rasgos de la fonología del tapiete. Aborda las reglas fonológicas que regulan las variaciones alofónicas y la disposición secuencial de fonemas en la sílaba y la palabra, a través de un estudio de las tendencias que subyacen a la co-ocurrencia de los segmentos en el marco de una palabra de tipo CV.CV. Fenómenos suprasegmentales tales como la armonía nasal, el acento, la estructura silábica y la reduplicación son también considerados. Específicamente, se discuten las variaciones morfo-fonológicas producidas por la armonía nasal y las consecuencias que estas variaciones acarrean sobre la estructura silábica y sobre el patrón de reduplicación parcial.

${ }^{1}$ Los datos para el presente artículo provienen del trabajo de campo realizado en el norte de Argentina, donde alrededor de 80 familias se encuentran asentadas en "Misión Los Tapietes", Tartagal, provincia de Salta. En consecuencia, esta descripción refleja la variedad hablada por los tapietes de esa región. Agradezco a los hablantes tapietes que colaboran con paciencia e interés en el trabajo de descripción de su lengua. Agradezco igualmente las sugerencias y correcciones de dos evaluadores anónimos que contribuyeron a mejorar el análisis de los datos. Cualquier error u omisión en el texto es mi responsabilidad.

2 Aparentemente, este no es el caso de los tapietes asentados en Samuwate, Bolivia, donde según testimonios de los tapietes de Tartagal, los niños aún hablan la lengua. La misma consideración es válida para los tapietes de Paraguay, donde la lengua goza de mayor vitalidad.

${ }^{3}$ La situación de la lengua tapiete en Tartagal y La Curvita merece un estudio sociolingüístico detallado. La Curvita es una comunidad multi-étnica donde los niños hablan español, al tiempo que preservan, en diferentes grados, alguna de las cuatro lenguas indígenas que ahí se hablan: wichí, toba, chorote y tapiete. Si bien es cierto que en Tartagal hay mayor presión para la adopción del español, los hablantes de la pequeña comunidad tapiete de La Curvita, aunque alejados de centros urbanos importantes, parecen estar abandonando la transmisión intergeneracional de la lengua. De esta manera, se observa una retracción en el uso por parte de los niños que no parecen usarla como medio de comunicación cotidiano. 


\section{FONOLOGÍA SEGMENTAL}

El tapiete posee 15 consonantes y 12 vocales, que se oponen sobre la base de la nasalidad: seis orales y seis nasales. La palabra fonológica se define como una raíz léxica con los clíticos asociados, los que se encuentran sujetos a reglas de asignación del acento. La Tabla y la Tabla 2 presentan el inventario de consonantes y de vocales tapietes. ${ }^{5}$

\begin{tabular}{|l|c|c|c|c|c|c|}
\hline & Bilabial & Alveolar & Palatal & Velar & Labio-velar & glotal \\
\hline Oclusiva sorda & $\mathrm{p}$ & $\mathrm{t}$ & & $\mathrm{k}$ & $\mathrm{k}^{\mathrm{w}}<\mathrm{kw}>$ & $\mathrm{P}<{ }^{\prime}>$ \\
\hline $\begin{array}{l}\text { Oclusiva sonora } \\
\text { prenasalizada }\end{array}$ & $\mathrm{mb}$ & $\mathrm{nd}$ & & $\mathrm{ng}$ & & \\
\hline Fricativa sorda & & $\mathrm{s}$ & $\int<\mathrm{sh}>$ & & & $\mathrm{h}$ \\
\hline Africada sorda & & & $\mathrm{t} \int<\mathrm{ch}>$ & & & \\
\hline Africada sonora & & & $\mathrm{d} 3<\mathrm{y}>$ & & & \\
\hline Vibrante simple & & $\mathrm{c}<\mathrm{r}>$ & & & & \\
\hline Aproximante & & & & & $\mathrm{W}$ & \\
\hline
\end{tabular}

Tabla 1. Consonantes del tapiete

\begin{tabular}{|l|c|c|c|}
\hline & Anterior & Central & Posterior \\
\hline Cerradas & $\mathrm{i} / \tilde{\mathrm{i}}<\mathrm{i}>$ & $\mathrm{z} / \tilde{\mathrm{x}}<\ddot{\mathrm{z}}>$ & $\mathrm{u} / \mathrm{u}<\ddot{\mathrm{u}}>$ \\
\hline Abiertas & $\mathrm{e} / \tilde{\mathrm{e}}<\ddot{\mathrm{e}}>$ & $\mathrm{a} / \tilde{\mathrm{a}}<\ddot{\mathrm{a}}>$ & $\mathrm{o} / \tilde{\mathrm{o}}<\ddot{\mathrm{o}}>$ \\
\hline
\end{tabular}

Tabla 2. Vocales del tapiete

${ }^{4}$ Abreviaturas: 1 'primera persona', 2 'segunda persona', 3 'tercera persona', AC 'activo', AUM 'aumentativo', C 'consonante', CAUS1 'causativo de verbos intransitivos', CAUS2 'causativo de verbos transitivos', DES 'desiderativo', DIM 'diminutivo', DIR 'direccional', EVD 'evidencial', EXCL 'exclusivo', FRC 'frecuentativo', FUT 'futuro', FUTDIST 'futuro distante', INCL 'inclusivo', INST 'instrumental', LOC 'locativo', LOCMOV 'locativo de movimiento', $\mathrm{N}$ 'consonante nasal', NEG 'negación', NOM 'nominalizador', ONH 'objeto no-humano incorporado', PAS 'pasado', PL 'plural', POS 'posesivo', REC 'pasado reciente', RES 'resultativo', SG 'singular, sil 'silábica', SUP 'superlativo', V 'vocal'.

${ }^{5}$ Los sonidos se transcriben en el Alfabeto Fonético Internacional (AFI) y los símbolos que se encuentran entre $<>$ representan el alfabeto tentativo tapiete. La fonología del tapiete se caracteriza por la armonía nasal, un proceso en virtud del cual las oclusivas sonoras pre-nasalizadas / $\mathrm{mb}$ nd $\mathrm{ng}$ ] y la africada sonora $/ \mathrm{d} 3 /$ tienen, en contextos nasales, una variante nasal $[\mathrm{m} \mathrm{n} \mathrm{n}]$ y [n], respectivamente (Cf. Sección 4). Este cuadro asume como oposición fundamental entre las obstruyentes el rasgo de la sonoridad; en consecuencia, se elige como representación fonológica la contraparte pre-nasalizada de la alternancia. Ver Costa $(2003,2007)$ para un análisis de la oposición entre obstruyentes basado en la sonoridad y Rose (2003) para un análisis basado en la existencia de un rasgo nasal que explicaría esta alternancia. 


\subsection{Descripción fonética de fonemas}

En esta sección se brinda la descripción fonética de las consonantes tapiete, que se ejemplifican en posición inicial y media de la palabra, ya que la lengua no acepta sílabas cerradas.

\subsubsection{Consonantes}

Las consonantes del tapiete son:

/p/: oclusiva bilabial sorda; ocurre en posición inicial y media

$\begin{array}{llll}\text { /pete/ } & \text { [pete] } & \text { pete } & \text { 'castiga' } \\ \text { /hupi/ } & \text { [hupi] } & \text { hupi } & \text { 'alza' }\end{array}$

/t/: oclusiva alveolar sorda; ocurre en posición inicial y media

$\begin{array}{llll}/ \text { taso/ } & {[\text { taso }]} & \text { taso } & \text { 'gusano' } \\ / \text { pota/ } & \text { [pota }] & \text { pota } & \text { 'quiere' }\end{array}$

/k/: oclusiva velar sorda; ocurre en posición inicial y media

$\begin{array}{llll}/ \mathrm{kiqa} / & {[\mathrm{kira}]} & \mathrm{kìa} & \text { 'sucio' } \\ / \mathrm{puku} / & {[\mathrm{puku}]} & \mathrm{puku} & \text { 'largo' }\end{array}$

$/ \mathbf{k}^{\mathrm{w}} /$ : oclusiva labiovelar sorda; ocurre en posición inicial y media

\begin{tabular}{|c|c|c|}
\hline /kªrasi / & [kwarasì] & \\
\hline
\end{tabular}

/R/: oclusiva glotal; ocurre en posición media ${ }^{6}$
/ka?a/
[ka?a]
ka'a
'bosque'

/mb/: oclusiva sonora bilabial pre-nasalizada; ocurre en posición inicial y media
/mberu/
[mberu]
mberu
'mosca'
/kambu/
[kambu]
kambu
'ser amamantado'

/nd/: oclusiva sonora alveolar pre-nasalizada; ocurre en posición inicial y media

$\begin{array}{llll}\text { /nde/ } & \text { [nde }] & \text { nde } & \text { 'tu' } \\ \text { /hendu/ } & {[\text { hendu }]} & \text { hendu } & \text { 'escucha' }\end{array}$

/gg/: oclusiva velar sonora pre-nasalizada; ocurre en posición media languza/ [anguza] anguya 'ratón'

${ }^{6}$ La oclusiva glotal también se presenta en posición inicial y final; sin embargo, en esos contextos no es fonémica (Cf. Sección 2.3.1). 
GonZÁLEZ: UnA APROXIMACión A LA FONOLOGÍA DEL TAPIETE (TuPÍ-GUARANí)

/s/: fricativa alveolar sorda; ocurre en posición inicial y media
/so?o/
[so?o]
so'o
'carne'
/hasa/
[hasa]
hasa
'pasa'

/S/: fricativa palatal sorda; ocurre en posición inicial y media

$\begin{array}{llll}\text { /Sure/ } & {[\text { ure] }} & \text { shure } & \text { 'papa' } \\ \text { /heJa/ } & {\left[\text { he } \int \mathrm{a}\right]} & \text { hesha } & \text { 've' }\end{array}$

/h/: fricativa glotal sorda; ocurre en posición inicial y media
/hal
[ha]
ha
'pelo'
/t̃ihe/
[tihe]
tihë
'cuela'

/t $\int /:$ africada palatal sorda; ocurre en posición inicial y media ${ }^{7}$
/dzot $\int a /$
[dzotfa]
yocha
'atrapa'

/d3/: africada palatal sonora; ocurre en posición inicial y media

$\begin{array}{llll}\text { /dzoio/ } & \text { [dzo?o }] & \text { yo'o } & \text { 'cava' } \\ \text { /adzuru/ } & {[\text { azuru }]} & \text { ayuru } & \text { 'loro' }\end{array}$

/w/: aproximante labio-velar; ocurre en posición inicial y media

$\begin{array}{llll}\text { /wera/ } & \text { [wera] } & \text { wera } & \text { 'brillante' } \\ \text { /awati/ } & \text { [awati] } & \text { awati } & \text { 'maíz' }\end{array}$

/r/: vibrante simple sonora alveolar; ocurre en posición inicial y media

$\begin{array}{llll}\text { /raha/ } & \text { [raha] } & \text { raha } & \text { 'lleva' } \\ \text { /here/ } & \text { [here] } & \text { here } & \text { 'llame' }\end{array}$

\subsubsection{Vocales}

El tapiete distingue entre vocales orales y nasales

/i/: vocal oral alta anterior no redondeada

[iro/ iro] 'amargo'

/1/: vocal nasal alta anterior no redondeada

$$
\pi_{1} /[1] \text { [i } \quad \ddot{i} \text { 'ser o estar' }
$$

/ì): vocal oral alta central no redondeada
/主/
[i்]
主
'agua'

/t/: vocal nasal alta no redondeada central
/K̃̃?

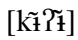
kï’
'ají'

${ }^{7}$ Cf. Sección 2.3.10 para la aparición de esta consonante en otros contextos. 
/e/: vocal oral media anterior no redondeada

$$
\text { /heta/ [heta] heta 'mucho' }
$$

/ê/: vocal nasal media anterior no redondeada
/hẽl
[hẽ]
hë
'salir'

/a/: vocal oral baja anterior no redondeada

lawara/ [awara] awara 'zorro'

/ã/: vocal nasal baja anterior no redondeada
/kãwi/

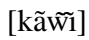
käwï
'chicha'

/u/: vocal oral alta posterior redondeada
/huri/
[huri]
huri
'ocho'

/ü/: vocal nasal alta posterior redondeada
/hũwa/
[hũ $\tilde{w}$ ]
hüwä
'negro'

/o/: vocal oral media posterior redondeada
/dzoka/
[dzoka]
yoka
'rompe'

/õ/: vocal nasal media posterior redondeada

/põra/ [põ̃ã] pörä 'hermoso'

\subsection{Lista de pares mínimos}

Si bien los pares mínimos no son absolutamente indispensables para establecer contrastes fonológicos, su presencia ayuda significativamente a la confirmación de la existencia de fonemas. La siguiente es una lista no exhaustiva de pares mínimos. Cuando es posible, se contrastan los segmentos en función de los rasgos fonéticos pertinentes que los oponen (por ejemplo, punto de articulación bilabial vs. alveolar para las oclusivas sordas), en posición inicial y media.

\subsubsection{Consonantes}

\begin{tabular}{|c|c|c|c|}
\hline /pẽku/ & [penku] & penku & 'tipo de pájaro' \\
\hline /tẽku/ & [teyku] & tenku & 'lengua de alguien' \\
\hline /dz主p主/ & [d $3 \dot{z} \mathrm{p} \dot{\mathrm{i}}]$ & $\mathrm{y} \dot{\mathrm{i}} \mathrm{p} \dot{\mathrm{i}}$ & 'tronco' \\
\hline /dzìti主/ & [d3i主主] & y主t主 & 'línea’ \\
\hline
\end{tabular}


GonZÁLEZ: UnA APROXIMACión A LA FONOLOGÍA DEL TAPIETE (TuPÍ-GUARANí)

\begin{tabular}{|c|c|c|c|c|}
\hline \multirow[t]{5}{*}{$/ \mathbf{k} /$} & /kw/ & \multirow[b]{2}{*}[\mathrm{karu}]{} & \multirow[b]{2}{*}{ karu } & \multirow[b]{2}{*}{ 'come' } \\
\hline & /karu/ & & & \\
\hline & $/ \mathrm{k}^{\mathrm{w}}$ aru/ & [kwaru] & kwaru & 'orina' \\
\hline & /saka/ & [saka] & saka & 'atado de leña' \\
\hline & $/ \mathrm{sak}^{\mathrm{w} a} /$ & [sakwa] & sakwa & 'agujero' \\
\hline \multirow[t]{3}{*}{$/ \mathbf{P} /$} & $/ \mathbf{k} /$ & & & \\
\hline & /he?a/ & [he?a] & he'a & 'mezcla' \\
\hline & /heka/ & [heka] & heka & 'busca' \\
\hline \multirow[t]{3}{*}{$/ P /$} & vs. $\quad / \mathbf{r} /$ & & & \\
\hline & $/ \mathrm{k \grave {i }} ? \ddot{\mathfrak{t}} /$ & {$[\mathrm{kì} ? \dot{\mathrm{t}}]$} & $k \dddot{k} \dddot{\prime} \dddot{t}$ & 'ají' \\
\hline & /kìrt/ & {$[\mathrm{kìr} \dot{\mathrm{i}}]$} & k̈̈r̈̈ & '(tiene) cosquillas' \\
\hline \multirow[t]{5}{*}{$/ \mathrm{t} /$} & vs. $\quad / \mathbf{r} /$ & & & \\
\hline & & {$[\mathrm{tu}]$} & $\mathrm{tu}$ & 'padre' \\
\hline & & {$[\mathrm{ru}]$} & ru & 'trae' \\
\hline & /pete/ & [pete] & pete & 'castiga' \\
\hline & /pere/ & [pere] & pere & 'herida' \\
\hline \multirow[t]{3}{*}{$/ \mathrm{t} \int /$} & $/ \mathbf{k} /$ & & & \\
\hline & /dzotfa/ & {$[\mathrm{d} z \circ \mathrm{f} \mathrm{a}]$} & yocha & 'atrapa' \\
\hline & /dzoka/ & [dzoka] & yoka & 'rompe' \\
\hline \multirow[t]{5}{*}{$/ \mathbf{s} /$} & vs. /S/ & & & \\
\hline & /sirririt/ & [strstrsit] & siririt & 'circula' \\
\hline & /Stritrit/ & {$\left[\int \dot{t r} \dot{t r} \dot{t}\right]$} & shirrirt & 'fríe' \\
\hline & /hesa/ & [hesa] & hesa & ‘su ojo’' \\
\hline & /he $\int \mathrm{a} /$ & [he $\left.\int \mathrm{a}\right]$ & hesha & 've' \\
\hline \multirow[t]{5}{*}{$/ \mathbf{d}_{3} /$} & vs. $/ \int /$ & & & \\
\hline & /dzuka/ & [dzuka] & yuka & 'mata' \\
\hline & /Suka/ & [Suka] & shuka & 'tipo de juego' \\
\hline & /hedza/ & [heza] & heya & 'deja' \\
\hline & /he $\int \mathrm{a} /$ & [he $\left.\int \mathrm{a}\right]$ & hesha & 've' \\
\hline \multirow[t]{5}{*}{$/ \mathbf{d}_{\mathbf{3}} /$} & vs. & & & \\
\hline & $/ \mathrm{d} 3^{\dot{t}} /$ & [dzit] & $y \dot{\mathrm{t}}$ & 'hacha' \\
\hline & $/ \mathrm{sì} /$ & {$[\mathrm{sit}]$} & sí & 'madre' \\
\hline & /kidze/ & [kizze] & kise & 'miedo' \\
\hline & /kise/ & [kise] & kise & 'cuchillo' \\
\hline
\end{tabular}


14

$/ \mathrm{mb} / \quad$ vs. $/ \mathbf{p} /$

$\begin{array}{llll}/ \text { tumba/ } & \text { [tumba] } & \text { tumba } & \text { 'huérfano' } \\ \text { /tupa/ } & \text { [tupa] } & \text { tupa } & \text { 'cama' } \\ \text { /mbite/ } & \text { [mbite] } & \text { mbite } & \text { 'en el medio' } \\ \text { /pite/ } & \text { [pite] } & \text { pite } & \text { 'besa' }\end{array}$

/nd/ vs. /t/

/ndirete/

/tirete/

/henda/

/heta/

/yg/ vs. /k/

/punga/

/puka/

[punga]

[puka]

punga

puka

[wit?a]

[pi?a]

[owa]

[opa] wì'a

pía

owa

opa /pria/

lopa/

\subsubsection{Vocales}

/e/ vs. /i/

$\begin{array}{llll}\text { /pere/ } & \text { [pere] } & \text { pere } & \text { 'herida' } \\ \text { /pire/ } & \text { [pire] } & \text { pire } & \text { 'piel' } \\ \text { /d3ãpe/ } & \text { [nampe] } & \text { ñampe } & \text { 'chueco' } \\ \text { /d3ãpi/ } & \text { [nampi] } & \text { ñampi } & \text { 'se afeita' }\end{array}$

$/ \mathbf{u} / \quad$ vs. /o/

$\begin{array}{llll}\text { /dzuhe/ } & \text { [dzuhe } & \text { yuhe } & \text { 'desea' } \\ \text { /dzohe/ } & {[\text { dzohe }]} & \text { yohe } & \text { 'lava' } \\ \text { /dzapu/ } & \text { [dzapu }] & \text { yapu } & \text { 'miente' } \\ \text { /dzapo/ } & \text { [dzapo] } & \text { yapo } & \text { 'hace' }\end{array}$

/a/ vs. /e/

$\begin{array}{llll}\text { /ha/ } & \text { [ha] } & \text { ha } & \text { 'va' } \\ \text { /he/ } & \text { [he] } & \text { he } & \text { 'su nombre' } \\ \text { /wata/ } & \text { [wata] } & \text { wata } & \text { 'camina' } \\ \text { /wate/ } & \text { [wate] } & \text { wate } & \text { 'arriba' }\end{array}$


GonZÁlEZ: UnA APROXIMACIÓN A LA FONOLOGÍA DEL TAPIETE (TuPí-GUARANÍ)

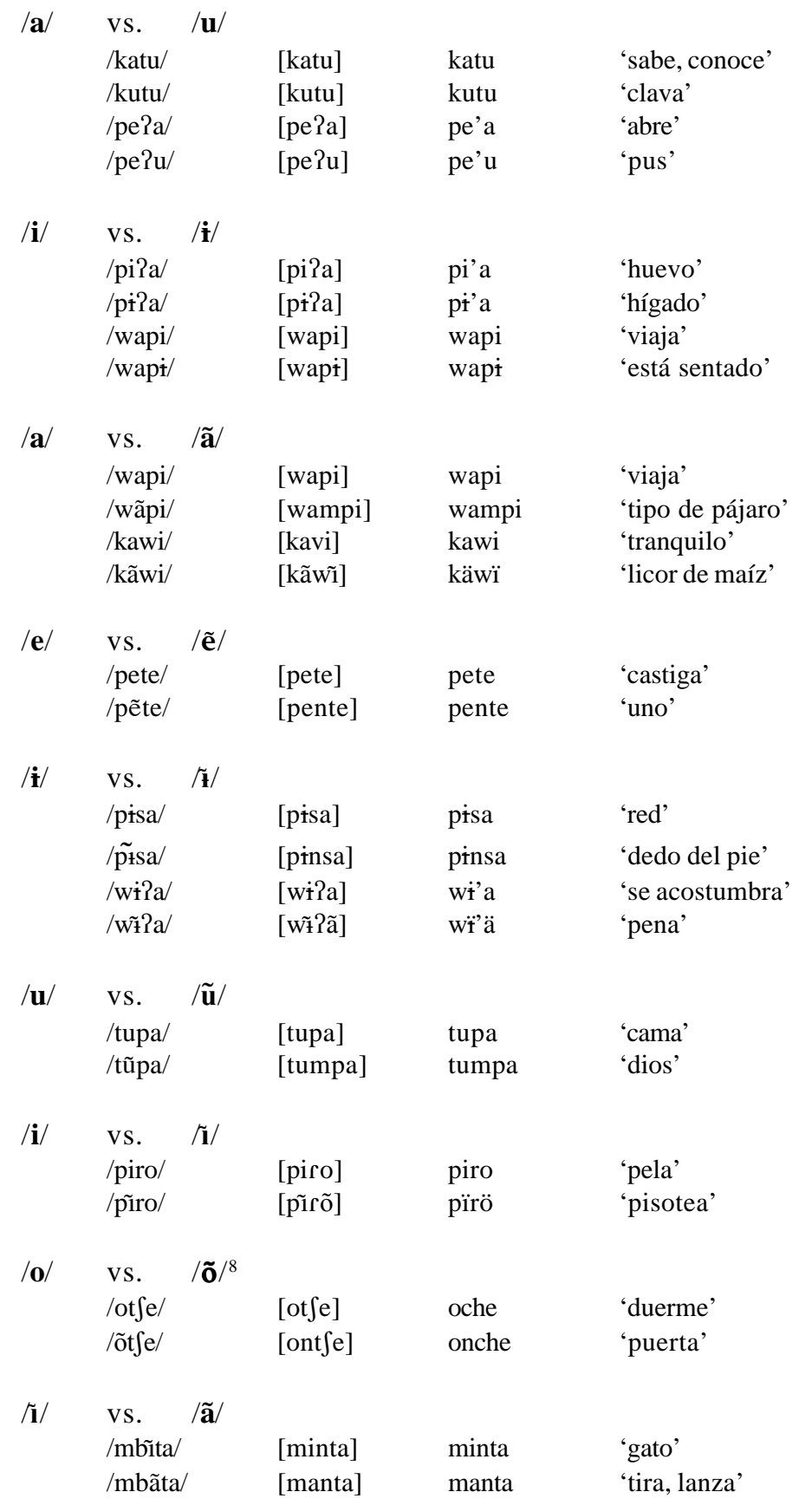

\footnotetext{
${ }^{8}$ Esta oposición tiene muy bajo peso funcional.
} 


$$
\text { vs. } / \tilde{\mathbf{a}} /
$$

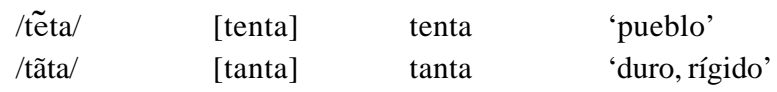

\subsection{Variaciones alofónicas}

Algunos segmentos presentan variaciones alofónicas; la siguiente discusión se limita a aquellos que no sufren cambios como consecuencia de la armonía nasal, la cual se abordará en la Sección 4.

\subsubsection{Inserción de la oclusiva glotal [?] en posición final e inicial de enunciado}

Una oclusiva glotal [?] se inserta en (i) posición final del enunciando, cuando la vocal final no es una vocal central [ì] (1) y en (ii) posición inicial del enunciando, cuando precede a una vocal (2).

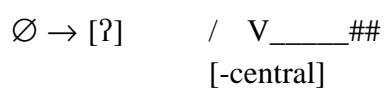

(2) $\varnothing \rightarrow[?] \quad /$ \#\#_ V

(3) [?ambeupo Seme haj 3uka huwi hẽẽ?]

a-mbe'u-po she-me ha'e yuka huwi hë'ë

1SGAC-contar-FUT 1SGPOS-marido ella/él mata sangre dulce

'Voy a contar que la diabetes mató a mi esposo'

\subsubsection{Inserción de la fricativa velar $[x]$ en posición final}

Una fricativa velar sorda se inserta en posición final del enunciado cuando la vocal final es la alta central [i] (4).

$$
\varnothing \rightarrow[\mathrm{x}] \quad / \quad \mathrm{V} \quad \ldots \#
$$

(5) $\quad / \mathrm{kt} /$

$[\mathrm{kix}]$

$\mathrm{ki}$

'piojo'

\subsubsection{Fricativización de /dz/}

La africada post-alveolar/dz/ se convierte en una fricativa post-alveolar [3] en posición intervocálica.

(6) $\left[\mathrm{d}_{3}\right] \rightarrow[3] \rightarrow / \mathrm{V}-\mathrm{V}$

(7) langudza/ [angúza] anguya 'ratón' 
En posición inicial de palabra (8) y después de una vocal nasal subyacente (9), esta consonante preserva su calidad africada.

$\begin{array}{llll}\text { (8) } / \text { dzadzu/ } & {[\mathrm{d} z a ́ z u} & \text { yayu } & \text { 'cuello' } \\ \text { (9) } / \text { mĩdze/ } & {[\text { míndze }]} & \text { minye } & \text { 'mete' }\end{array}$

\subsubsection{Elisión de la oclusiva glotal [?]}

En lenguaje informal, la oclusiva glotal es elidida cuando se encuentra en posición intervocálica (10).
(10) $[?] \rightarrow[\varnothing] / V_{-} \mathrm{V}$
(11) $/$ mboRe/ [mbó?e] $\rightarrow \quad$ [mbóe] mbo'e 'enseña'

languia/ [angúia] $\rightarrow \quad$ [angúa] angu'a 'mortero'

\subsubsection{Palatalización}

En lenguaje informal, la vocal media no redondeada [e] y la vocal alta central [í se realizan como una aproximante palatal [j] cuando son precedidas por una vocal baja acentuada [a] en posición final de palabra (12).

(12) $[\mathrm{e} i \mathrm{i}] \rightarrow[\mathrm{j}] /$
\[ \begin{array}{l}\text { +baja } \\ \text { +acento }\end{array} \]

Esta regla debe ser precedida por la regla de elisión de la oclusiva glotal en posición intervocálica para que la variación encuentre el contexto apropiado (13).
(13) /mbaie/
[mbá?e $] \rightarrow$
[mbáe] $\rightarrow$ [mbáj]
mba'e
'cosa' /sãdza?t/
[sandzáì̀] $\rightarrow$
[sandzáti $\rightarrow$
[sandzáj]
sanya'i
'niño/a'

\subsubsection{Elisión de la vocal final}

Una vocal final es elidida cuando la oclusiva glotal precedente es eliminada y la vocal que precede a la oclusiva glotal es de la misma calidad vocálica (14). Esta regla se aplica después de la regla de elisión de la oclusiva glotal en posición intervocálica (15) ${ }^{9}$.

${ }^{9}$ Las consecuencias que esta regla tiene para la silabificación y el lugar del acento se consideraran en la Sección 5.1. 
(14)

$\begin{array}{ll}\text { Elisión de la oclusiva glotal } & \mathrm{CV}_{1} ? \mathrm{~V}_{1} \\ \text { Elisión de la vocal final } & \mathrm{CV}_{1} \mathrm{~V}_{1} \text { ? } \\ \mathrm{CV}_{1}\end{array}$

(15) /kapi?i/

$[$ kapíii $] \rightarrow[$ kapíi $] \rightarrow$ kapí $]$

kapi'i

'pasto'

\subsubsection{Palatalización de [i]}

La vocal alta central [ít tiende a palatalizarse después de las consonantes palatales [ $\left.\int\right]$

(16) $y\left[d_{3}\right](17)$.

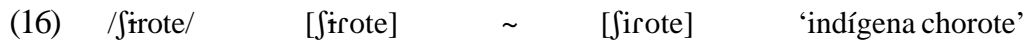

(17) $/ \mathrm{d}_{3}$ ípi/ $\quad\left[\mathrm{d}_{3}\right.$ ẗpi $] \sim$ [dzipi $]$ 'yípi'

\subsubsection{Palatalización de [s]}

La fricativa palatal sorda [ $\left.\int\right]$ es un alófono de la fricativa sorda alveolar [s] después de la vocal anterior [i].

(18)

$[\mathrm{s}] \quad \rightarrow$

$\left[\int\right] /$

[V]

$\left[\begin{array}{l}+ \text { anterior } \\ + \text { alta }\end{array}\right]$

(19)

$\begin{array}{llll}\text { /tiwisa/ } & \text { [tißíja } & \text { tiwisha } & \text { 'ancho' } \\ \text { /istmba/ } & \text { [if́́mba } & \text { ishimba } & \text { 'huérfano' }\end{array}$

En algunos casos, la fricativa alveolar sorda [s] ocurre después de la vocal anterior [i] (20).

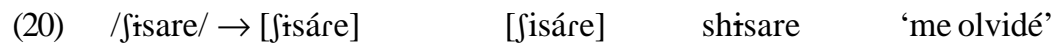

No obstante, esta vocal debe analizarse como una vocal central alta /it/ subyacente que se manifiesta como una vocal anterior [i], como consecuencia de haber sido palatalizada por la fricativa palatal sorda precedente.

\subsubsection{Distribución complementaria de $[\beta]$ y [w]}

La fricativa sonora bilabial [ $\beta$ ] y la semivocal labio-velar [w] están en distribución complementaria: $[\mathrm{w}$ ] se encuentra tanto en posición inicial de palabra como en posición intermedia, mientras que $[\beta]$ sólo se encuentra en posición intervocálica, donde nunca es precedida o seguida por la vocal central alta [i] (21). 
$\begin{array}{llllll}(21)[\mathrm{w}] & \rightarrow \quad[\beta] \quad / \quad & \mathrm{V} & \mathrm{V} \\ & & & \end{array}$

Ejemplos de esa distribución se ofrecen en (22) y (23).

(22) Alófono [w]

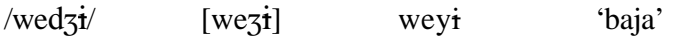

litwit [ítit] itwi tierra'

(23) Alófono [ $[\beta]$

$\begin{array}{llll}\text { /tiwika/ } & {[\mathrm{ti} \beta \mathrm{ikwa}]} & \text { tiwikwa } & \text { 'el ano de alguien' } \\ \text { /huwi } \mathrm{a} \text { / } & {[\mathrm{hu \beta ifa}]} & \text { huwisha } & \text { 'su cacique' } \\ \text { /kawi/ } & {[\mathrm{ka \beta i}]} & \text { kawi } & \text { 'bien' } \\ \text { /owa/ } & {[\mathrm{o} \beta \mathrm{a}]} & \text { owa } & \text { 'seis' } \\ \text { /wewe/ } & {[\text { weße] }} & \text { wewe } & \text { 'vuela' } \\ \text { /hekowe/ } & {[\text { hekoße] }} & \text { hekowe } & \text { 'su vida' }\end{array}$

\subsubsection{Distribución complementaria de [k] y [t $\left.\int\right]$}

La africada palatal sorda [t $\left.\int\right]$ es un alófono de la oclusiva velar sorda /k/ antes de las vocales anteriores [i] y [e] (24).

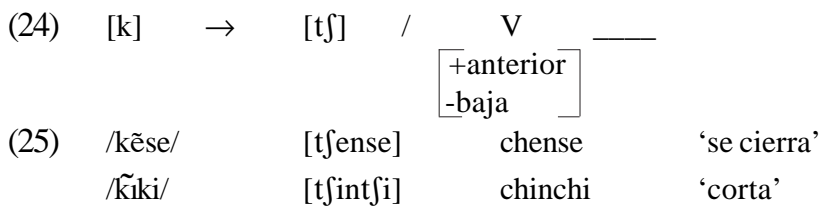

No obstante, se observan ejemplos en los que la oclusiva velar sorda [k] se encuentra antes de las vocales anteriores [i] y [e]. En esos casos, la oclusiva velar sorda [k] está siempre precedida por una vocal no-anterior [u o a] (26). Por su parte, la africada palatal sorda $\left[\mathrm{t} \int\right]$ nunca ocurre antes de las vocales no-anteriores $[\dot{\mathrm{t}}],[\mathrm{o}] \mathrm{y}[\mathrm{u}]^{10}$.

${ }^{10}$ Otras lenguas guaraníes como el avá-guaraní (chiriguano) y el guaraní paraguayo exhiben una oclusiva velar sorda [k] en el mismo contexto, es decir, antes de vocales anteriores [i] y [e] (Dietrich 1986; Canese 1983). Al parecer, en tapiete la distribución complementaria de una oclusiva velar sorda $[\mathrm{k}]$ antes de vocales no-anteriores y de una africada palatal sorda [ $\left.\mathrm{t} \int\right]$ antes de vocales anteriores es un rasgo innovador. En los préstamos recientes del español, sin embargo, se ha constatado la presencia de la oclusiva velar sorda [k] antes de las vocales anteriores [i] y [e].

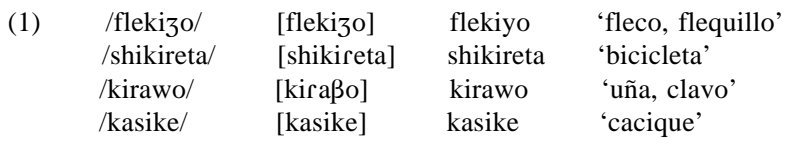


20

(26)
LIAMES 8

$\begin{array}{ll}\text { sapuke } & \text { 'grito' } \\ \text { sapupuke } & \text { 'está gritando' } \\ \text { iyuke'i } & \text { 'cuñada de la mujer' } \\ \text { tìroke } & \text { 'corral, gallinero' } \\ \text { síwa oke } & \text { 'frente quemada' } \\ \text { Taki } & \text { 'Taki' }\end{array}$

Para resumir, la africada palatal sorda ocurre antes de las vocales anteriores [i] y [e], nunca se encuentra antes de las vocales no anteriores [i] , [o] y [u], donde aparece la oclusiva velar sorda $[\mathrm{k}]$. No obstante, $\left[\mathrm{t} \int\right]$ y [k] contrastan antes de la vocal baja [a], ya que hay, al menos, un par mínimo que opone esas dos consonantes (27). Esta oposición nunca ocurre en posición inicial de la palabra y no parece muy funcional en la fonología del tapiete.

$$
\begin{array}{llll}
\left./ \text { dzot } \int a\right] & {\left[\text { dzot } \int a\right]} & \text { yocha } & \text { 'atrapa' } \\
/ \text { dzoka/ } & {[\text { dzoka }]} & \text { yoka } & \text { 'rompe' }
\end{array}
$$

\subsubsection{Metátesis}

En lenguaje informal, la fricativa glotal [h] intercambia su posición con la vocal siguiente cuando se encuentra en una sílaba no acentuada (28).
(28) $\mathrm{hV} \rightarrow \mathrm{Vh} \quad / \mathrm{V}$
$\left[\begin{array}{l}\text { +sílaba } \\ \text {-acento }\end{array}\right] \quad\left[\begin{array}{l}\text { +sílaba } \\ \text { +acento }\end{array}\right]$
(29) /wãhe [wãh] wähë 'llega'

\subsubsection{Variación libre de $[\mathrm{n}] \sim[\mathrm{r}]$}

En contexto nasal, la vibrante simple [ $\mathrm{r}$ ] e realiza como un nasal alveolar [n] o como una vibrante simple nasalizada [r] (30).

$$
/ \mathrm{r} / \rightarrow[\mathrm{n}] \sim[\tilde{\mathrm{r}}] / \quad \underset{[+ \text { nasal }]}{\mathrm{V}}-
$$

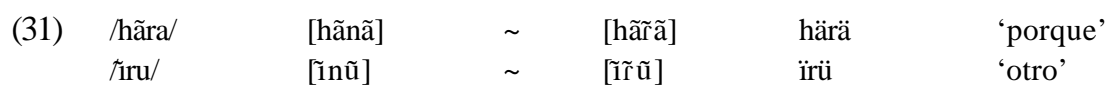

\section{FONOTÁCTICA}

En esta sección se examina la frecuencia, la distribución y las reglas de co-ocurrencia de los segmentos en la sílaba y la palabra. 


\subsection{Frecuencia y distribución de los segmentos}

Las oclusivas sordas [p], [t], [k] y [?] son las consonantes más frecuentes en el corpus. Todas las consonantes, a excepción de la oclusiva glotal [? $]^{11}$ y de la oclusiva sonora velar pre-nasalizada [ng], pueden ocurrir en posición inicial de palabra. La fricativa glotal [h], las oclusivas sordas [p], [t], [k] y la africada sonora [dz] son las consonantes que más frecuentemente ocupan la posición de $\mathrm{C}_{1}$ dentro de una palabra de tipo CV.CV. Además, todas las consonantes pueden ocurrir en posición inicial de sílaba. Las consonantes que ocurren más frecuentemente en esta posición son la oclusiva glotal [?], las oclusivas bilabial y alveolar [p] y [t], y la aproximante labiovelar [w]. La Tabla 3 muestra la frecuencia de los segmentos sobre una base de 382 palabras de tipo CV.CV. Las consonantes se presentan por orden de frecuencia.

\begin{tabular}{|c|c|c|c|c|c|}
\hline Segmento & $\mathrm{C}_{1}$ & $\%$ & \begin{tabular}{|l|} 
Segmento \\
\end{tabular} & $\mathrm{C}_{2}$ & $\%$ \\
\hline $\mathrm{h}$ & 61 & 16 & ? & 57 & 15 \\
\hline $\mathrm{p}$ & 56 & 15 & $\mathrm{t}$ & 47 & 12 \\
\hline $\mathrm{t}$ & 49 & 13 & $\mathrm{p}$ & 42 & 11 \\
\hline $\mathrm{d}_{3}$ & 44 & 11.5 & $\mathrm{~W}$ & 37 & 10 \\
\hline $\mathrm{k}$ & 38 & 10 & $\mathrm{k}$ & 34 & 9 \\
\hline $\mathrm{mb}$ & 24 & 6 & $\mathrm{~S}$ & 25 & 6.5 \\
\hline $\mathrm{m}$ & 23 & 6 & $\mathrm{~h}$ & 16 & 4 \\
\hline $\mathrm{W}$ & 23 & 6 & $\mathrm{n}$ & 13 & 3.4 \\
\hline $\mathrm{n}$ & 19 & 5 & $d_{3}$ & 12 & 3.1 \\
\hline $\mathrm{s}$ & 12 & 3 & $\mathrm{~m}$ & 11 & 2.8 \\
\hline $\mathrm{n}$ & 9 & 2 & nd & 10 & 2.6 \\
\hline $\mathrm{r}$ & 6 & 1.5 & $\mathrm{mb}$ & 8 & 2 \\
\hline $\int$ & 5 & 1.3 & $\mathrm{n}$ & 8 & 2 \\
\hline $\mathrm{t} \int$ & 5 & 1.3 & $\int$ & 7 & 1.8 \\
\hline nd & 3 & 0.7 & $\mathrm{kw}$ & 5 & 1.3 \\
\hline$\eta$ & 1 & 0.3 & gg & 5 & 1.3 \\
\hline$?$ & 0 & 0 & r & 4 & 1 \\
\hline 1g & 0 & 0 & t5 & 3 & 0.8 \\
\hline
\end{tabular}

Tabla 3. Frecuencia de consonantes en una palabra de tipo CV.CV

\subsection{Co-ocurrencias de las consonantes}

Dentro de una raíz léxica de tipo CV.CV se observa una tendencia general de las consonantes a la disimilación respecto al punto de articulación. Las posibles combinaciones

${ }^{11}$ En la Sección 2.3.1 se mostró que la oclusiva glotal puede ocurrir en posición inicial y final del enunciado, donde no contrasta con ningún otro segmento. 
de consonantes se presentan en la Tabla 4, donde se ve que las oclusivas sordas se combinan frecuentemente entre ellas y con la oclusiva glotal. Sin embargo, se observa una tendencia a evitar la co-ocurrencia de dos oclusivas sordas producidas en el mismo punto de articulación dentro de una palabra de tipo CV.CV (i.e. es más común que una oclusiva bilabial sorda [p] co-ocurra con una oclusiva alveolar sorda [t] o con una oclusiva velar sorda [k] que con otra oclusiva bilabial sorda). De la misma manera, se observa una tendencia de la alveolar fricativa sorda [s] y de la palatal fricativa sorda [ $]$ ] a co-ocurrir más frecuentemente con las oclusivas sordas evitando la combinación mutua. Esto también se observa con la fricativa glotal [h], la cual solo co-ocurre con una palatal fricativa sorda [ $]$ ] y con otra fricativa glotal [h] en sólo una palabra respectivamente, aunque su co-ocurrencia con la fricativa alveolar [s] es muy frecuente.

En cuanto a la africada palatal sonora [d3], la combinación se da principalmente con las oclusivas sordas, con la fricativa alveolar sorda [s] y con la fricativa glotal [h] pero sólo en una palabra se combina con otra africada palatal sorda [t $\left.\int\right]$ y con una africada palatal sonora [d3].

\begin{tabular}{|c|c|c|c|c|c|c|c|c|c|c|c|c|c|c|c|c|c|c|c|}
\hline $\mathrm{C} 2$ & $\mathrm{p}$ & $\mathrm{t}$ & $\mathrm{k}$ & $\mathrm{k}^{\mathrm{w}}$ & $?$ & $\mathrm{~S}$ & $\int$ & $\mathrm{h}$ & $\mathrm{t} \int$ & $\mathrm{d}_{3}$ & I & W & $\mathrm{mb}$ & nd & 1]g & $\mathrm{m}$ & $\mathrm{n}$ & $\eta$ & $\mathrm{n}$ \\
\hline $\mathrm{p}$ & $\mathrm{X}$ & $\mathrm{X}$ & $\mathrm{X}$ & $\mathrm{X}$ & $\mathrm{X}$ & $\mathrm{X}$ & $\mathrm{X}$ & $\mathrm{X}$ & & $\mathrm{X}$ & $\mathrm{X}$ & $\mathrm{X}$ & & & $\mathrm{X}$ & & $\mathrm{X}$ & & $\mathrm{X}$ \\
\hline $\mathrm{t}$ & $\mathrm{X}$ & $\mathrm{x}$ & $\mathrm{X}$ & $\mathrm{X}$ & $\mathrm{X}$ & $\mathrm{X}$ & & & & $\mathrm{X}$ & $\mathrm{X}$ & $\mathrm{X}$ & $\mathrm{X}$ & $\mathrm{x}$ & $\mathrm{x}$ & $\mathrm{X}$ & $\mathrm{X}$ & & \\
\hline $\mathrm{k}$ & $\mathrm{X}$ & $\mathrm{X}$ & & & $\mathrm{x}$ & $\mathrm{X}$ & & & & $\mathrm{x}$ & $\mathrm{X}$ & $\mathrm{X}$ & $\mathrm{X}$ & $\mathrm{x}$ & & $\mathrm{X}$ & $\mathrm{x}$ & & $\mathrm{X}$ \\
\hline $\mathrm{k}^{\mathrm{w}}$ & $\mathrm{X}$ & & & $x$ & & & & & & & $x$ & $\mathrm{X}$ & & & & & & & \\
\hline \multicolumn{20}{|l|}{$?$} \\
\hline $\mathrm{S}$ & $\mathrm{X}$ & & $\mathrm{x}$ & $\mathrm{X}$ & & $\mathrm{x}$ & & & & & $\mathrm{X}$ & $\mathrm{X}$ & & & $\mathrm{x}$ & $\mathrm{x}$ & & & \\
\hline $\int$ & $\mathrm{X}$ & $\mathrm{x}$ & $\mathrm{x}$ & & $\mathrm{X}$ & & & & & & $\mathrm{X}$ & & & & & & & & \\
\hline $\mathrm{h}$ & $\mathrm{X}$ & $\mathrm{X}$ & $\mathrm{X}$ & $\mathrm{X}$ & $\mathrm{X}$ & $\mathrm{X}$ & $\mathrm{X}$ & $\mathrm{X}$ & & $\mathrm{X}$ & $\mathrm{X}$ & $\mathrm{X}$ & & $\mathrm{x}$ & $\mathrm{X}$ & $\mathrm{X}$ & $\mathrm{X}$ & & $\mathrm{X}$ \\
\hline $\mathrm{t} \int$ & & & & & & $\mathrm{X}$ & & & $\mathrm{X}$ & & $X$ & & & & & & $\mathrm{X}$ & & \\
\hline $\mathrm{d}_{3}$ & $\mathrm{X}$ & $\mathrm{x}$ & $\mathrm{X}$ & & $\mathrm{X}$ & $\mathrm{X}$ & $x$ & $\mathrm{X}$ & $\mathrm{X}$ & $\mathrm{X}$ & $X$ & $\mathrm{X}$ & & & & & & & \\
\hline I & & $\mathrm{X}$ & $\mathrm{X}$ & & $\mathrm{X}$ & & & $\mathrm{X}$ & & $\mathrm{X}$ & $\mathrm{X}$ & $\mathrm{X}$ & & & & & & & \\
\hline $\mathrm{W}$ & $\mathrm{X}$ & $\mathrm{X}$ & $\mathrm{x}$ & & $\mathrm{X}$ & $\mathrm{X}$ & $\mathrm{X}$ & $\mathrm{X}$ & & $\mathrm{X}$ & $\mathrm{X}$ & $\mathrm{X}$ & & & & & & & \\
\hline $\mathrm{mb}$ & $\mathrm{X}$ & $\mathrm{X}$ & $\mathrm{x}$ & & $\mathrm{x}$ & $\mathrm{x}$ & & $\mathrm{X}$ & & $\mathrm{x}$ & $\mathrm{X}$ & $\mathrm{X}$ & & & & $\mathrm{x}$ & & & \\
\hline nd & & & & & $\mathrm{X}$ & & $\mathrm{X}$ & & & & & $\mathrm{X}$ & & & & & & & \\
\hline \multicolumn{20}{|l|}{ 1g } \\
\hline $\mathrm{m}$ & $\mathrm{X}$ & $\mathrm{X}$ & $\mathrm{X}$ & & $\mathrm{x}$ & & & $\mathrm{X}$ & & $\mathrm{X}$ & $\mathrm{X}$ & $\mathrm{X}$ & $\mathrm{X}$ & $\mathrm{X}$ & & & $\mathrm{X}$ & & $\mathrm{X}$ \\
\hline $\mathrm{n}$ & $\mathrm{X}$ & $\mathrm{X}$ & & & $\mathrm{X}$ & & & $\mathrm{X}$ & & & & & $\mathrm{X}$ & & $\mathrm{X}$ & $\mathrm{X}$ & $\mathrm{X}$ & & \\
\hline$\eta$ & & $\mathrm{X}$ & & & & & & & & & & & & & & & & & \\
\hline $\mathrm{J}$ & $\mathrm{X}$ & $\mathrm{X}$ & & & $\mathrm{X}$ & & & $\mathrm{X}$ & & & & $\mathrm{X}$ & & $\mathrm{X}$ & & $\mathrm{X}$ & $\mathrm{X}$ & & $\mathrm{X}$ \\
\hline
\end{tabular}

Tabla 4. Co-ocurrencias de consonantes en una palabra CV.CV 


\section{ARMONÍANASAL}

La nasalidad es un rasgo prosódico, suprasegmental, característico de la fonología de las lenguas tupí-guaraníes, cuyo dominio y modalidad varía de acuerdo a las características particulares de cada lengua. De una manera general, se puede decir que la armonía nasal crea alternancias consonánticas: en entornos nasales, las consonantes nasales son alófonos de las oclusivas sonoras pre-nasalizadas y de las oclusivas sordas; en tanto, las oclusivas sonoras pre-nasalizadas y las oclusivas sordas se encuentran en entornos orales ${ }^{12}$.

El tapiete, como otras lenguas de esta familia, se caracteriza por su armonía nasal, un proceso por el cual una raíz léxica nasal expande su nasalidad afectando a vocales y consonantes adyacentes. Como consecuencia de la armonía nasal, las oclusivas sonoras pre-nasalizadas /mb nd $\mathrm{ng} /$ y la africada sonora / $\mathrm{d} 3 /$ tienen, en contextos nasales, una variante nasal [m n $]$ y [n], respectivamente. Por su parte, las oclusivas sordas [p t k kw], la africada palatal sorda $\left[\mathrm{t} \int\right]$ y las fricativas sordas [ $\mathrm{s} \int$ ] presentan una variante pre-nasalizada [mp nt $\eta \mathrm{k} \mathrm{nk}^{\mathrm{w}}$ ], [nt $\left.\int\right]$ y [ns nf], respectivamente, cuando se encuentran después de una vocal nasal. En la sección siguiente, se analizan las reglas que gobiernan la armonía nasal en tapiete, abordando específicamente el elemento que dispara la nasalidad, el dominio sobre el cual se difunde, la dirección de esa difusión y los elementos que la bloquean.

\subsection{Elementos que disparan la nasalidad}

Como se dijo anteriormente, en tapiete, la nasalidad es un rasgo suprasegmental que se expande a partir de la sílaba nasal acentuada de una raíz léxica y, de esta manera, afecta la calidad de las vocales y consonantes que pertenecen a las sílabas que le son adyacentes, traspasa los límites del morfema y crea alteraciones vocálicas y consonánticas ${ }^{13}$.Una sílaba nasal se define como una sílaba acentuada que contiene (i) una vocal nasal y/o (ii) una consonante nasal. Si bien las vocales que siguen o preceden una consonante nasal tienden a ser nasalizadas, su realización fonética es de una vocal apenas nasalizada o completamente oral (32). Por el contrario, las vocales nasales acentuadas seguidas o precedidas de consonantes orales son fuertemente nasalizadas $(33)^{14}$.

${ }^{12}$ El fenómeno de la armonía nasal en las lenguas tupí-guaraníes ha sido discutido en diferentes trabajos. Algunos de ellos lo han abordado en el marco de la descripción de una lengua de la familia (Costa 2003, Dietrich 1986, Dooley 1984; Fargetti 2001; Ferreira 1981; Guedes 1991; Gregores y Suárez 1967; Harrison y Taylor 1971, Nascimento 2008, Rose 2001-2002); otros lo han hecho desde un punto de vista teórico analizando los alcances de diferentes propuestas teóricas para una explicación satisfactoria del fenómeno. Ver Barratt (1981) para una crítica al enfoque autosegmental en guaraní; Picanço (2005) para un enfoque de la fonología del mundurukú en el marco de la Teoría de la Optimalidad y Costa (2007) para un recapitulativo comprensivo de diferentes aproximaciones a la armonía nasal en guaraní.

${ }^{13}$ Las consecuencias que la nasalidad tiene a nivel morfo-fonológico se examinan en la Sección 7.1.5.

${ }^{14}$ Un fenómeno similar es reportado por Gregores y Suárez (1967:67) para el guaraní. 


$\begin{array}{llll}\text { (32) } / \text { ndõhe/ } & {[\text { nõhẽ] } \sim \text { nnohe }]} & \text { nohë } & \text { 'saca' } \\ \text { (33) } / \text { wẽ?e/ } & \text { [wẽ?ẽ] } & \text { wë'ë } & \text { 'vomita' }\end{array}$

El dominio de la nasalidad en tapiete es la palabra fonológica definida como una unidad prosódica. Dado que la nasalidad es desencadenada solamente por una sílaba nasal acentuada, la oposición oral-nasal es funcional sólo en ese ámbito. En otras palabras, la calidad oral-nasal de los segmentos adyacentes está determinada por la naturaleza oral-nasal de la sílaba acentuada de la raíz léxica. En consecuencia, la nasalidad no es contrastiva en las sílabas adyacentes ${ }^{15}$.

\subsubsection{Segmentos sordos pre-nasalizados}

Además de las consonantes sonoras pre-nasalizadas, el tapiete presenta consonantes sordas pre-nasalizadas. En realidad, la secuencia de una vocal seguida de una consonante nasal antes de una obstruyente sorda constituye una variación alofónica de una vocal nasal. Es decir, una secuencia como [VNC] es la forma superficial de una vocal nasal subyacente seguida de una obstruyente sorda. En estos casos la consonante nasal es homorgánica a la consonante sorda siguiente, la vocal nasal subyacente es fonéticamente realizada como una vocal oral y la porción nasal de la consonante sorda pre-nasalizada es claramente perceptible; algunos ejemplos se muestran en $(34)^{16}$.

\begin{tabular}{|c|c|c|c|}
\hline /ãpo/ & [ampo] & ampo & 'este' \\
\hline /pẽte/ & [pente] & pente & 'uno' \\
\hline /dzãkì/ & [naykí] & $\tilde{\text { nankì }}$ & 'mojado' \\
\hline /hãse/ & [hanse] & hanse & 'grita' \\
\hline /mbi] $\mathrm{i} /$ & {$\left[\min \int \mathrm{i}\right]$} & minshi & 'chico' \\
\hline /sãza?ł̇/ & [sanzaì̇] & sanya't & 'niño' \\
\hline
\end{tabular}

En el habla formal, la calidad oral de la vocal y la clara articulación de la consonante nasal son fácilmente perceptibles (35), mientras que la articulación de una vocal nasal con o sin una consonante nasal que la siga es común en el discurso informal (36).

$\begin{array}{llll}\text { /pẽte/ } & \text { [pente] } & \text { pente } & \text { 'uno' } \\ \text { /pẽte/ } & {[\text { pẽnte] } \sim[\text { [pẽte] }} & \text { pente } & \text { 'uno' }\end{array}$

Además, la consonante fricativa palatal sorda [ $\left.\int\right]$ y la africada palatal sonora [d3] muestran también un grado de pre-nasalización después de una vocal nasal (37).

${ }^{15}$ El tapiete se distingue, así, de lenguas como, por ejemplo, el emerillón (Rose 2001-2002:169) o el juruna (Farguetti 2001:105), lenguas en las que el dominio de la nasalidad es el morfema y, en consecuencia, una palabra puede estar compuesta de un morfema oral y un morfema nasal.

16 Priest (1980:10) reporta para el sirionó una distribución similar de la consonante alveolar sorda pre-nasalizada [ $\left.{ }^{\mathrm{n}} \mathrm{t}\right]$ que funciona como alófono /t/ después de vocal nasal. 


$\begin{array}{llll}\text { (37) } / \mathrm{mbi}[\mathrm{i} / & {\left[\min \int \mathrm{i}\right]} & \operatorname{minshi} & \text { 'pequeño' } \\ / \mathrm{mbid} z \mathrm{e} / & {[\text { mindze }]} & \text { minye } & \text { 'meter' }\end{array}$

Sin embargo, la articulación de la consonante nasal homorgánica que precede a la fricativa palatal sorda es mucho más inestable; así, es común encontrar una vocal completamente nasal en este contexto (38).

(38) $/ \mathrm{mbi}\left[\mathrm{i} / \quad\right.$ [mĩ $\left.\int \mathrm{i}\right] \quad$ minshi 'pequeño'

\subsection{Sentido de la expansión de la nasalidad}

La nasalidad se expande regresiva y progresivamente y se bloquea bajo condiciones específicas que se discuten a continuación.

\subsubsection{Expansión regresiva de la nasalidad}

La nasalidad se expande a partir de la raíz nasal hacia su izquierda afectando la calidad de las consonantes y de las vocales precedentes. Como se dijo anteriormente, la nasalidad se expresa ya sea a través de una vocal nasal (por ejemplo, [hãrãyga] hä'änga 'similar') (39) o a través de una sílaba acentuada cuya estructura fonética es de tipo CVN (por ejemplo, [kampémpe] kampempe 'totora') (40). En (40), la penúltima sílaba nasal acentuada es responsable de la expansión regresiva de la nasalidad que afecta la calidad de la sílaba precedente, la cual se vuelve nasal. Los dos tipos de sílabas nasales acentuadas expanden su nasalidad regresivamente.

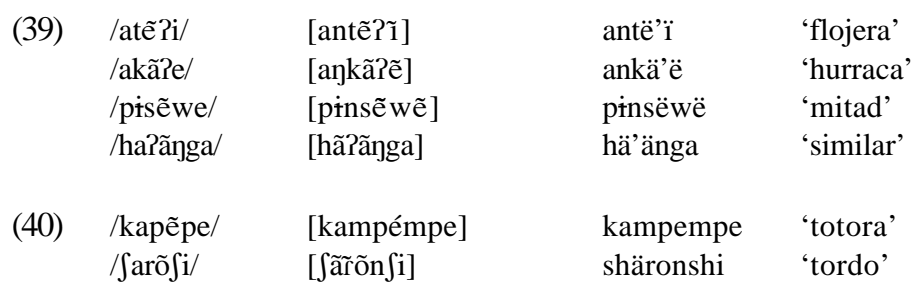

Finalmente, las consonantes sonoras pre-nasalizadas de la sílaba acentuada de una raíz léxica (por ejemplo, [mbóPe] mbo'e 'enseñar'), también desencadenan la expansión de la nasalidad. Este fenómeno que afecta la forma de los prefijos se discute en la sección 7.1.5.1.

\subsubsection{Expansión progresiva de la nasalidad}

En el ámbito de la palabra fonológica, la nasalidad se expande progresivamente a través de la oclusiva glotal [?], la vibrante simple [r], la aproximante labiovelar [w] y la 
fricativa glotal [h] afectando la calidad de la última vocal (41). En consecuencia, la calidad nasal de la vocal final no es fonémica en este contexto ${ }^{17}$.

\begin{tabular}{|c|c|c|c|c|}
\hline & \multirow{4}{*}{$\begin{array}{l}\text { 1mbã?e/ } \\
\text { /wãhe/ } \\
\text { /kãre/ } \\
\text { /hõwi/ }\end{array}$} & [mã?ẽ] & mä'ë & 'mira' \\
\hline & & [wãhẽ] & wähë & 'llega' \\
\hline & & [kãrẽ] & kärë & 'se rasca' \\
\hline & & [hõwĩ] & höwï & estrecho \\
\hline
\end{tabular}

\subsubsection{Segmentos que bloquean la expansión de la nasalidad}

Las obstruyentes sordas bloquean la expansión progresiva de la nasalidad en el ámbito de la palabra fonológica. Así, dada una raíz léxica nasal, su última vocal es oral si está precedida ya sea por una oclusiva (42), una africada (43) o una fricativa (44).

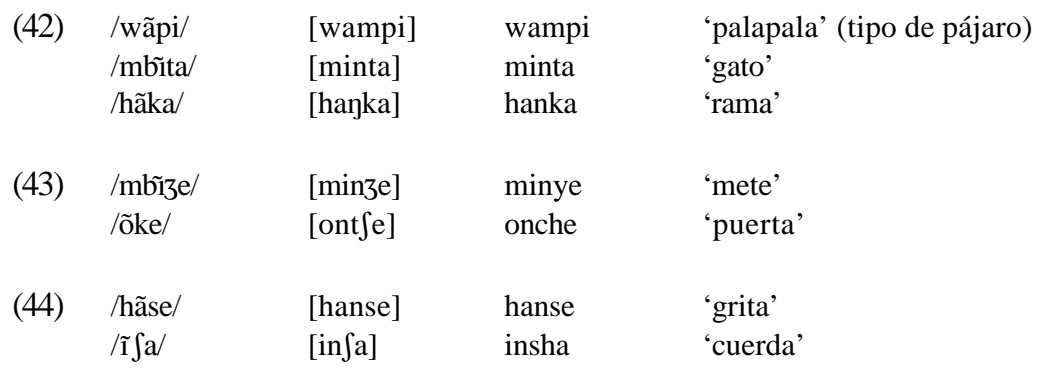

\section{ACENTO}

En tapiete el acento es predecible y recae en la penúltima sílaba de la palabra (45).

$\begin{array}{lll}\text { (45) } & \text { tata }] & \text { 'fuego' } \\ {[\text { dza.só.za }]} & \text { yasoya } & \text { 'olla' }\end{array}$

Este patrón acentual puede variar como consecuencia de la concatenación de ciertos morfemas. En (46) y (47), la sufijación del morfema aspectual -ma 'RES' y del morfema diminutivo - $m i$ 'DIM' respectivamente, agrega una sílaba a la palabra sin alterar el lugar del acento, lo que resulta en la creación de una palabra esdrújula.

\begin{tabular}{|c|c|c|c|}
\hline (46) & $\begin{array}{l}\text { [ká.ru] } \\
\text { [ká.ru.ma] }\end{array}$ & $\begin{array}{l}\text { karu } \\
\text { káruma }\end{array}$ & $\begin{array}{l}\text { 'come' } \\
\text { 'comió' }\end{array}$ \\
\hline 47) & 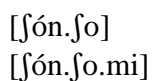 & $\begin{array}{l}\text { shonsho } \\
\text { shónshomi }\end{array}$ & $\begin{array}{l}\text { 'tipo de pájaro' } \\
\text { 'tipo de pájaro' }\end{array}$ \\
\hline
\end{tabular}

${ }^{17}$ La capacidad de estos segmentos [j], [w], [h], [?] y [r] para dejar pasar la nasalidad se documenta también en otras lenguas de la familia (Borella 2000:56; Costa 2003:84; Nascimento 2008:17). 


\subsection{Variaciones del lugar del acento}

Algunas de las reglas propuestas en la Sección 2.3 para explicar las alternancias fonológicas tienen consecuencias con respecto al lugar del acento en la palabra. En particular, la interacción entre las reglas de supresión de oclusiva glotal (48) y de supresión de vocal final (49) resulta en palabras cuyo patrón de acentuación se desvía del patrón canónico. Como se vio anteriormente, la regla de supresión de vocal final elide una vocal final cuando la oclusiva glotal precedente es elidida y la vocal que precede la oclusiva glotal es de la misma calidad. Esta regla se aplica después de la regla de supresión de la oclusiva glotal.

$$
\begin{array}{cl}
{[?] \rightarrow[\varnothing] / V_{-}} & \mathrm{V} \\
\mathrm{V} \rightarrow[\varnothing] / \quad & \mathrm{V} \\
& {[\alpha \text { alta }] \_ \text {\# }} \\
& {[\beta \text { anterior }]}
\end{array}
$$

La aplicación de estas reglas modifica significativamente la silabificación de las palabras y su patrón acentual, lo que se puede apreciar especialmente en las palabras trisilábicas. Así, debido a la eliminación de la sílaba final, una palabra trisilábica se vuelve disilábica y, puesto que el lugar del acento no se ve alterado, la forma resultante es una palabra disilábica oxítona (50).

$$
\text { /kapißi/ [kapíî] } \rightarrow \text { [kapíi] } \rightarrow \text { [kapí] } \rightarrow \quad \text { kapi'i } \quad \text { 'pasto' }
$$

\section{ESTRUCTURA DE LASÍLABA}

El tapiete posee sílabas abiertas. Aunque también se observan sílabas fonéticamente cerradas, éstas son el resultado de procesos fonológicos (por ejemplo, armonía nasal) y morfo-fonológicos (por ejemplo, supresión de la vocal). La estructura de la sílaba se enta en (51).

(51)

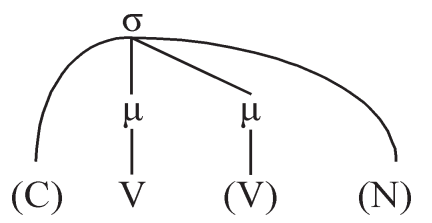

En la sección siguiente, se examinan los tipos de estructura silábica que se distinguen en tapiete junto con los tipos marginales que resultan de procesos fonológicos. 


\subsection{Tipos de estructura silábica}

El tapiete posee tres tipos de sílabas abiertas: CV, V y CVV; es decir, que las sílabas pueden tener un ataque, pero no aceptan una coda. ${ }^{18}$ Además, el núcleo de la sílaba puede constar de una o dos vocales. Los tipos más frecuentes son CV y V, seguidos por CVV (52).

\begin{tabular}{|c|c|c|c|c|}
\hline /he.pi/ & [hé.pi] & CV.CV & hepit & 'caro' \\
\hline /a.ra/ & [á.ra] & V.CV & ara & 'cielo' \\
\hline /hai.mbe/ & [háj.mbe] & CVV.CV & haimbe & 'tuesta' \\
\hline
\end{tabular}

\subsection{Secuencias de vocales}

Las secuencias de vocales que se observan en tapiete son realizadas fonéticamente como diptongos descendientes $\left[\mathrm{a}^{\mathrm{i}}\right],\left[\mathrm{e}^{\mathrm{i}}\right],\left[\mathrm{o}^{\mathrm{i}}\right],\left[\mathrm{a}^{\mathrm{u}}\right]$ y $\left[\mathrm{o}^{\mathrm{u}}\right](53)$.

\begin{tabular}{|c|c|c|c|}
\hline \multirow[t]{5}{*}{ (53) } & [háimbe] & haimbe & 'tuesta' \\
\hline & [éi] & ei & 'miel' \\
\hline & [mbói] & mboi & 'víbora' \\
\hline & {$\left[\int \mathrm{a}^{u}\right]$} & shau & 'ocho' \\
\hline & [ón] & ou & 'viene' \\
\hline
\end{tabular}

Sin embargo, desde el punto de vista fonológico, una secuencia de vocales puede ser interpretada como monosilábica o disilábica de acuerdo a la interacción entre el lugar del acento en la palabra y la distribución de los segmentos en la sílaba. Dado que el patrón acentual por defecto es que el acento caiga en la penúltima sílaba, las secuencias de vocales que siguen (54) o preceden (55) la sílaba acentuada deben analizarse como monosilábicas.

$\begin{array}{lllll}\text { (54) /mbiwei/ } & \text { [mbí.wej] } & \text { CV.CVV } & \text { mbiwei } & \text { 'tipo de pato' } \\ \text { (55) /taitetu/ } & \text { [taj.té.tu] } & \text { CVV.CV.CV } & \text { taitetu } & \text { 'chancho' }\end{array}$

Siguiendo el mismo criterio, es decir, el lugar del acento, una secuencia de vocales $\mathrm{V}_{1} \mathrm{~V}_{2}$ donde $\mathrm{V}_{1}$ está acentuada y ocurre antes de la última sílaba debe considerarse monosilábica.

\begin{tabular}{|c|c|c|c|}
\hline $\begin{array}{l}\text { /haimbe/ } \\
\text { /haiwi/ }\end{array}$ & $\begin{array}{l}\text { [háj.mbe] } \\
\text { [háj.wi] }\end{array}$ & $\begin{array}{l}\text { CVV.CV } \\
\text { CVV.CV }\end{array}$ & $\begin{array}{l}\text { haimbe } \\
\text { haiwi }\end{array}$ \\
\hline
\end{tabular}

\footnotetext{
${ }^{18}$ Como se verá en la Sección 6.4 existen excepciones a esta generalización
} 
Finalmente, una secuencia acentuada de vocales que ocurre en aislación (57) o en posición final de palabra se analiza como disilábica (58).

$\begin{array}{llllll}\text { (57) } & \text { /ei/ } & \text { [éj] } & \text { V.V } & \text { ei } & \text { 'miel' } \\ & & & & & \\ \text { 58) } & \text { /hambei/ } & \text { [haméj] } & \text { CV.CV.V } & \text { haméi } & \text { 'siempre' } \\ & \text { /mbundui/ } & \text { [mundúj] } & \text { CV.CV.V } & \text { mundúi } & \text { 'maní' } \\ & \text { /karai/ } & \text { [karáj] } & \text { CV.CV.V } & \text { karái } & \text { 'criollo' } \\ & \text { /kwimbae/ } & {[\text { kwimbáe] }} & \text { CV.CV.V } & \text { kwimbáe } & \text { 'hombre' } \\ \text { /kwatia/ } & {[\text { kwatía] }} & \text { CV.CV.V } & \text { kwatía } & \text { 'cuaderno' }\end{array}$

En conclusión, la lengua sólo permite la realización de sílabas abiertas, aunque se observan sílabas cerradas en contextos predecibles: así, en posición intermedia, una sílaba puede presentar como coda una consonante nasal que, fonológicamente, funciona como la manifestación de la nasalidad de la vocal nasal que la precede ${ }^{19}$. Además, el tapiete no permite la realización de secuencias de consonantes en el ámbito de una sílaba. Las únicas secuencias de consonantes que se observan se encuentran en contextos específicos que se limitan a (i) un conjunto de oclusivas pre-nasalizadas (sean estas consideradas como una unidad fonológica (por ejemplo, [anguza] /angudza/ 'ratón' o como el resultado de la expresión de la nasalidad vocálica (por ejemplo, [manki] /mãki/ 'mojó), y a (ii) la secuencia de la oclusiva velar sorda [k] y la semi-consonante labio-velar [w], las cuales, dadas las condiciones de distribución, deben analizarse como un solo segmento.

\subsection{Oclusivas sonoras pre-nasalizadas y consonantes africadas}

Aunque las consonantes sonoras pre-nasalizadas [mb], [nd] y [ng], y las consonantes africadas [t $\mathrm{f}]$ y [d3] constituyen segmentos articulatoriamente complejos, se analizan como segmentos simples porque despliegan la misma distribución silábica de los fonemas simples, es decir, sólo se encuentran en posición inicial de sílaba (59).

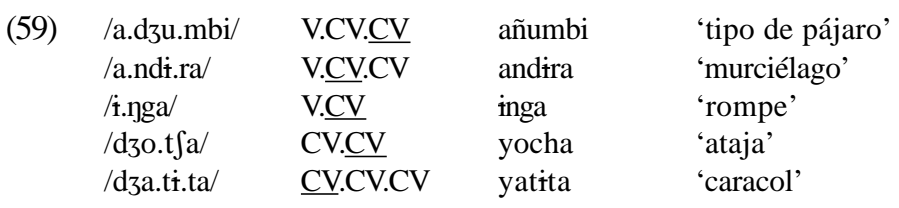

Con respecto a los segmentos prenasalizados, una distinción se observa entre las oclusivas bilabial [mb] y alveolar [nd] por un lado, y la oclusiva velar [ng], por el otro: esta última es mucho menos frecuente que su contraparte bilabial y alveolar y ocurre siempre en posición media, aunque no siempre en el límite de morfemas como se reporta en otras lenguas de la familia (Costa 2007:91).

${ }^{19}$ Otros contextos en los que se observan sílabas cerradas se describen en las Secciones 2.3.1, 2.3 .2 y 6.5 . 


\subsection{Oclusivas sordas prenasalizadas}

Las oclusivas sordas prenasalizadas no son fonemas de la lengua, ya que la porción nasal de estos segmentos fonéticamente complejos es la manifestación superficial de la nasalidad de la vocal que los precede. Es decir, una vocal fonológicamente nasal puedemanifestarse a través de (i) una vocal nasal plena (por ejemplo, [ẽ]) (60) y de una vocal oral seguida de una consonante nasal (por ejemplo, [en], [ej) (61). En consecuencia, las sílabas que contienen vocales nasales despliegan dos tipos de estructura silábica que se representan abajo.

(60)
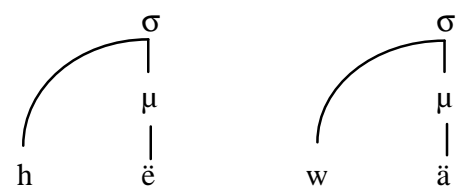

(61)

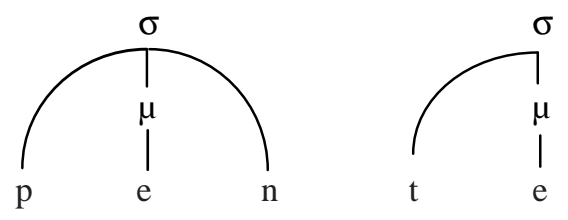

Otros tipos de estructuras silábicas (por ejemplo, VC) ocurren como resultado de las alternancias fonológicas que se discuten en la sección siguiente.

\subsection{Variaciones de la estructura silábica}

Además de los tipos de estructura silábica presentados en la sección anterior, se observa otro tipo de estructura silábica marginal que resulta de alternancias fonológicas. Específicamente, la regla de Metátesis es responsable del intercambio de posición entre la fricativa glotal $[\mathrm{h}]$ con la vocal siguiente cuando se encuentra en una sílaba no acentuada, como se formalizó en (28). La aplicación de esta regla resulta en la creación de una sílaba cerrada de tipo VC, que difiere del tipo canónico $(\mathrm{C}) \mathrm{V}(\mathrm{V})(\mathrm{N})$. En consecuencia, después de la aplicación de esta regla una palabra disilábica tal como $\left[\mathrm{C}_{1} \mathrm{~V}_{1} \cdot \mathrm{C}_{2} \mathrm{~V}_{2}\right]$ cuya $\mathrm{C}_{2}$ es una fricativa glotal se convierte en $\left[\mathrm{C}_{1} \mathrm{~V}_{1} \cdot \mathrm{V}_{2} \mathrm{C}_{2}\right](62)$.

(62) /wãhe/

$$
\begin{array}{lll}
\text { [wã.hẽ }] & \rightarrow & \text { [wã.ẽh] } \\
{\left[\mathrm{C}_{1} \mathrm{~V}_{1} \cdot \mathrm{C}_{2} \mathrm{~V}_{2}\right]} & \rightarrow & {\left[\mathrm{C}_{1} \mathrm{~V}_{1} \cdot \mathrm{V}_{2} \mathrm{C}_{2}\right]}
\end{array} \quad \text { wähë } \quad \text { 'llega' }
$$

En los casos en los que la estructura silábica es de tipo $\left[\mathrm{C}_{1} \mathrm{~V}_{1} \cdot \mathrm{C}_{2} \mathrm{~V}_{1}\right]$ con una fricativa glotal como $\mathrm{C}_{2}$ el patrón silábico resultante es de tipo $\left[\mathrm{C}_{1} \mathrm{~V}_{1} \mathrm{C}_{2}\right]$. Es decir, la secuencia de dos vocales de la misma calidad no redunda en el alargamiento de la vocal, sino que ésta se realiza como un segmento simple (63). 
GONZÁLEZ: UNA APROXIMACIÓN A LA FONOLOGÍA DEL TAPIETE (TuPí-GUARANÍ)

(63) $/ \mathrm{raha} /[\mathrm{raha}] \quad \rightarrow \quad[\mathrm{raah}] \quad \rightarrow \quad[\mathrm{rah}] \quad$ raha 'llega' $\left[\mathrm{C}_{1} \mathrm{~V}_{1} \cdot \mathrm{C}_{2} \mathrm{~V}_{1}\right] \quad \rightarrow \quad\left[\mathrm{C}_{1} \mathrm{~V}_{1} \cdot \mathrm{V}_{1} \mathrm{C}_{2}\right] \quad \rightarrow \quad\left[\mathrm{C}_{1} \mathrm{~V}_{1} \mathrm{C}_{2}\right]$

Así, en virtud de la aplicación de las reglas fonológicas, el único segmento permitido en posición de coda junto con las consonantes nasales es la fricativa glotal [h].

\section{MORFOFONOLOGÍA}

En esta sección, se examinarán las variaciones alomórficas que resultan de procesos morfofonológicos, a saber, las alternancias fonológicas que ocurren en las uniones de morfemas, las alternancias que resultan de la armonía nasal, las alternancias en la ubicación del acento y la reduplicación parcial.

\subsection{Variaciones alomórficas}

\subsubsection{Elisión de $a$}

Como se dijo anteriormente, una fricativa glotal $[\mathrm{h}]$ puede intercambiar su posición con la vocal precedente. El mismo proceso ocurre en la frontera morfemática entre una raíz léxica y el marcador de persona - $h a$ 'EXCL': cuando la última vocal de la raíz léxica es una vocal anterior baja [a], se elide la vocal del sufijo.

(64)

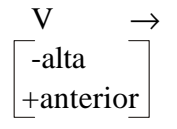

$\varnothing /$

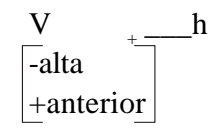

Este proceso tiene consecuencias para la posición del acento, ya que la palabra resultante se vuelve oxítona, como se ve en (65) donde iyeta-ha 'hablamos' sufre una modificación de su estructura silábica y de su acentuación, como consecuencia de la aplicación de las reglas expuestas.

$$
\begin{aligned}
& \text { /idzetáha/ [i.ze.tá.ha] } \rightarrow \text { [i.ze.tá.ah] } \rightarrow \text { [i.ze.táh] 'hablamos' } \\
& \text { [V.CV.CV.CV] } \rightarrow \text { [V.CV.CV.VC] } \rightarrow \text { [V.CV.CVC] }
\end{aligned}
$$

\subsubsection{Cerramiento de [e] y palatalización de [i]}

La vocal anterior [e] del sufijo -se 'DES' se realiza como una vocal anterior alta [i] cuando está precedida por una sílaba acentuada y seguida por una vocal baja anterior [a] (66), después de lo cual, la vocal resultante se transforma en una aproximante palatal [j] (67). Una ejemplificación de este proceso se presenta en (68). 
(66)

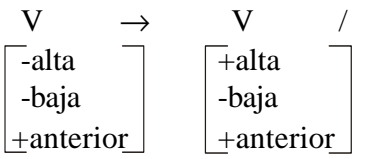

$\mathrm{V}$

[+acento] $\left.\begin{array}{l}\mathrm{V} \\ \text {-alta } \\ \text { +baja } \\ \text { +anterior }\end{array}\right]$

(67)

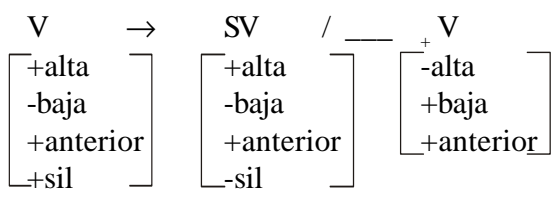

(68) $/$ karuháseape/ $\rightarrow$ [karuháseape $] \rightarrow[$ karuhásiape $] \rightarrow[$ karuhásjape $]$

karu - há - se - a - pe

comer-EXCL-DES-NOM-LOC

comedor (lit. lugar donde siempre se come)

\subsubsection{Coalescencia}

En lenguaje informal, el marcador de la tercera persona $o$ - y el verbo $\ddot{\imath}$ 'ser' se fusionan en una vocal nasal simple $\ddot{e}$. La vocal resultante combina las características de las vocales adyacentes originales (69): comparte con el fonema /i/ el rasgo [+anterior] [+nasal], y con el marcador de la tercera persona $o$ - el rasgo [-alta] (70).

(69)

$$
\left.\stackrel{\left[\begin{array}{l}
\text { +posterior } \\
\text {-alta } \\
\text {-baja } \\
\text { +oral }
\end{array}\right]}{+}\left[\begin{array}{l}
\mathrm{V} \\
\text { +anterior } \\
\text { +alta } \\
\text { +nasal }
\end{array}\right] \quad \begin{array}{l}
\mathrm{V} \\
\text { +anterior } \\
\text {-alta } \\
\text {-baja } \\
+ \text { nasal }
\end{array}\right]
$$

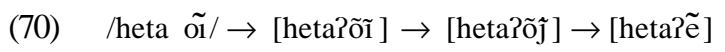

a. heta o-i

mucho $3 \mathrm{AC}$-ser

hay mucho

b. heta $\ddot{e}$

mucho 3:ser

hay mucho

\subsubsection{Alternancias de $m b \dot{t} \sim m \dot{t}$ 'CAUS1'}

El prefijo causativo $m b \dot{t} \sim m \dot{t}$ 'CAUS1' muestra las siguientes alternancias:

(i) La vocal central [i] se transforma en vocal anterior cuando precede a una consonante palatal (71). 
(71)

a. /midzedzẽ?e/ [minenẽrẽ]

mì-ñeñë'ë

CAUS1-sonar repetidamente

produce un sonido repetidamente

b. /mikiki/ [mint $\left.\int i n t \int i\right]$

min-chinchi

CAUS1-cortar

hacé que (alguien) corte

(ii) La vocal central [i] experimenta un proceso de armonización cuando la primera vocal de la raíz léxica a la cual está unida es una vocal redondeada posterior [u] $(72)^{20}$.

(72)

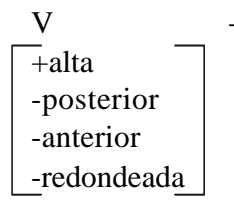

(73)

a. /mıpũa/

mum-pü'ä

CAUS1-levantar

levantar (algo)

b. /mbıpupu/

mbu-pupu

CAUS1-hervir

hacé(lo) hervir

[mumpũ̃ã]

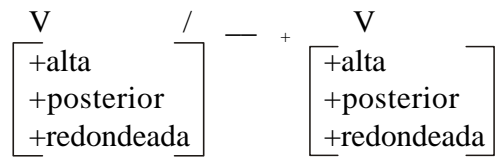

[mbupupu]

En este punto es interesante notar que la forma cognada de este prefijo en otras lenguas tupí-guaraníes es mbo- mo- 'CAUS1'y, en consecuencia, el tapiete parece haber retenido el rasgo [+ posterior] de la vocal del prefijo en el contexto especificado en $(72)^{21}$. En ese sentido hay dos raíces verbales $h \ddot{e}$ 'salir' y che 'dormir' que no se combinan con $m b \dot{t}-\sim m \dot{t}-$, sino con las formas originales $m b o-\sim m o-(74)$. Probablemente, estas raíces hayan sido tomadas del avá-guaraní (chiriguano) como una unidad inanalizable.

${ }^{20}$ Dietrich (1986:75) reporta la misma variante $m b u$ - $\sim m u$ - para el avá-guaraní (chiriguano) aunque el contexto de realización difiere del contexto tapiete: la variante $m b u$ - $\sim m u$ - aparece delante de consonante palatal.

${ }^{21}$ Entre otras, el avá-guaraní (chiriguano) (Dietrich 1986) y el guaraní paraguayo (Gregores y Suárez 1967) son lenguas que presentan las formas mbo- mo-. 
(74)

a. mo-hë

CAUS1- salir

(lo) hace salir

b. mo-nye

CAUS1- dormir

(lo) hace dormir

(iii) La vocal central [i] se elide cuando se sufija a una raíz cuyo primer segmento es una $\operatorname{vocal}(75)$.

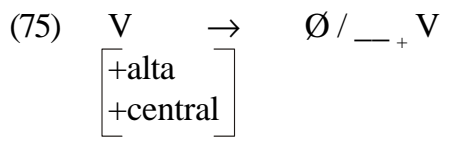

(76)
a. $/$ mbiodjil/ $\quad[$ mbo3i $]$
mb-oyí
CAUS1-cocer
(lo) hace cocer

b. /mbiidzeta/ [mbizeta]

a-mb-iyeta

1SGAC-CAUS1-hablar

(lo) hago hablar

(iv) Las raíces verbales que comienzan con una fricativa glotal [h] sufren las siguientes alternancias cuando están precedidas por el prefijo causativo $m b \dot{i}-$ :

La fricativa glotal [h] se elide (77), después de lo cual se aplica la regla de eliminación de $\dot{i}$ (75). Esta alternancia es común en hablantes jóvenes (78).

(77) $\begin{aligned} & \mathrm{C}_{1} \rightarrow \\ & \left.\begin{array}{l}+ \text { glotal } \\ + \text { fricativa }\end{array}\right]\end{aligned}$

$\varnothing /$ CAUS $1_{+}$

(78)

a. $\quad$ /ambihakupota/ $\rightarrow$ [ambihakupota] $\rightarrow$ [ambiakupota] $\rightarrow$ [ambakupota]

a-mbi-haku-pota

1SGAC-CAUS1-caliente-FUT

voy a calentar(lo) 
b. /ambihedzaka/

[ambihedzaka] $\rightarrow$ [ambizaka]

a-mbi-heya-ka

1SGAC-CAUS1-dejar-CAUS2

hago que lo deje

\subsubsection{Armonía nasal}

En la sección 4, se examinaron los efectos de la difusión de la nasalidad en el ámbito de las raíces léxicas. En esta sección, se presentan las alternancias morfo-fonológicas que resultan de la armonía nasal, es decir, las formas alternativas exhibidas por los afijos como consecuencia de su adyacencia a una raíz oral o nasal.

\subsubsection{Variaciones alomórficas debidas a la difusión regresiva de la nasalidad}

Las alternancias morfo-fonológicas que resultan de la difusión regresiva de la nasalidad son desencadenadas por los siguientes segmentos encontrados en sílabas acentuadas: (i) una vocal nasal, (ii) una consonante nasal, y (iii) una consonante sonora prenasalizada.

Las consonantes sonoras prenasalizadas tienen la particularidad de desencadenar la nasalidad, sólo regresivamente. En otras palabras, la porción nasal de las consonantes prenasalizadas expande la nasalidad hacia atrás, mientras que la porción oral de esos segmentos preserva la calidad oral de los segmentos siguientes; es decir, no hay difusión progresiva de la nasalidad ${ }^{22}$. Esto se ejemplifica en (79), donde la oclusiva sonora prenasalizada [mb] de la raíz nasal mbo'e 'estudia' es responsable de la variante nasal del marcador de tercera persona (pe. $\tilde{n} \dot{t}=$ ' 3 '), mientras que las vocales siguientes son completamente orales ([mbõ?e]).

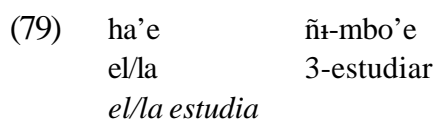

La Tabla 5 Tabla muestra ejemplos de las variaciones alomórficas de marcadores personales que resultan de la difusión regresiva de la nasalidad. Por cada raíz nasal se presenta una raíz oral, de manera que los cambios operados en los prefijos pueden apreciarse completamente.

\footnotetext{
${ }^{22}$ Un fenómeno similar ocurre en el kaiwá (Harrison y Taylor 1971:18).
} 


\begin{tabular}{|c|c|c|c|}
\hline & Categoría gramatical & Raíz léxica nasal & Raíz léxica oral \\
\hline Nasalidad vocálica & 1SGAC & $\begin{array}{l}\text { ä - hä'ä } \\
\text { 1SGAC-probar } \\
\text { probar (comida) }\end{array}$ & $\begin{array}{l}\text { a - pota } \\
\text { 1SGAC-querer } \\
\text { quiero }\end{array}$ \\
\hline \multirow[t]{2}{*}{ Alternancia $m \sim m b$} & Incorporación & $\begin{array}{l}\text { ma - moi - po } \\
\text { ONH-cocinar-FUT } \\
\text { cocinará algo }\end{array}$ & $\begin{array}{l}\text { mba - piwara } \\
\text { ONH-perforar } \\
\text { perfora algo }\end{array}$ \\
\hline & CAUS1 & $\begin{array}{l}\text { min - chinchi } \\
\text { CAUS1-cortar } \\
\text { hacelo cortar (con sierra) }\end{array}$ & $\begin{array}{l}\text { mbi - hakwa } \\
\text { CAUS1-puntudo } \\
\text { saca(le) punta }\end{array}$ \\
\hline \multirow[t]{2}{*}{ Alternancia $n \sim n d$} & 2SGPOS & $\begin{array}{l}\text { nt - membł } \\
\text { 2SGPOS-hijo de la mujer } \\
\text { Su hijo }\end{array}$ & $\begin{array}{l}\text { ndt - yiwa } \\
2 \text { SGPOS-brazo } \\
\text { su brazo }\end{array}$ \\
\hline & 1PLINCLPOS & $\begin{array}{l}\text { ñani - membi } \\
\text { IPLINCLPOS-hijo de la mujer } \\
\text { nuestros (incl.) hijos }\end{array}$ & $\begin{array}{l}\text { ñandt - yiwa } \\
\text { 1PLINCLPOS-brazo } \\
\text { muestros (incl.) brazos }\end{array}$ \\
\hline \multirow[t]{2}{*}{ Alternancia $\tilde{n} \sim y$} & $3 \mathrm{POS}$ & $\begin{array}{l}\tilde{\mathbf{n i}} \mathbf{\mathbf { i }}-\text { mbo't } \\
3 \text { POS-collar } \\
\text { su collar }\end{array}$ & $\begin{array}{l}\text { yi - wati } \\
\text { 3POS-maíz } \\
\text { su maíz }\end{array}$ \\
\hline & 1PLINCL & $\begin{array}{l}\text { ña - hë } \\
\text { 1PLINCL-salir } \\
\text { salimos (incl.) }\end{array}$ & $\begin{array}{l}\text { ya - raha } \\
\text { 1PLINCL-llevar } \\
\text { llevamos (incl.) }\end{array}$ \\
\hline
\end{tabular}

Tabla 5.Variaciones alomórficas debidas a la difusión regresiva de la nasalidad

\subsubsection{Difusión regresiva de la nasalidad y raíces monosilábicas}

El tapiete codifica gramaticalmente la oposición entre posesión alienable y posesión inalienable: es decir, las raíces monosilábicas que nombran las partes del cuerpo nunca ocurren de manera aislada, sino que deben estar siempre prefijadas por un marcador de posesión. Este hecho importa desde el punto de vista de la difusión regresiva de la nasalidad, ya que existen raíces léxicas nasales monosilábicas, que son fonéticamente orales, pero cuya calidad nasal se expresa a través del prefijo de posesión. Esto se ve claramente en (80) y (81): aunque las raíces kä 'pecho' y kü 'lengua' son fonéticamente orales, su cualidad nasal se expresa en la sílaba precedente a través de la variante nasal del marcador posesivo. En (80), la nasalidad regresiva emerge como una vocal nasal cuya manifestación fonética es del tipo VN; en (81), la nasalidad se manifiesta, además, a través de la variante nasal de la alternancia consonántica $n d \sim n$. En ambos casos, la vocal de la raíz permanece fonéticamente oral.

(80) /i kã]

[inka]

in-ka

3SG-pecho 'su pecho' 
(81) /nde kũ/

[nejku]

nen-ku

2SGAC-lengua 'su lengua'

\subsubsection{Variaciones alomórficas debido a la expansión progresiva de la nasalidad}

La expansión progresiva de la nasalidad puede traspasar los límites del morfema si el ataque silábico del morfema siguiente no contiene un segmento capaz de bloquear la nasalidad (es decir, una oclusiva, una fricativa o una africada). La nasalidad se extiende progresivamente en ese contexto cuando una oclusiva glotal [?], una vibrante simple [r], una labiovelar aproximante $[\mathrm{w}]$ o una fricativa glotal $[\mathrm{h}]$ ocupan la posición de ataque de la sílaba del morfema siguiente. En (82), el nominalizador -wa se vuelve nasal en virtud de la nasalidad progresiva que parte de la sílaba nasal acentuada [hã] y se expande gracias a la presencia de la consonante labiovelar aproximante [w].

(82) /hãwewa/

[hãwẽwã]

häwë-wa

opaco-NOM 'opaco'

\subsubsection{Prefijo causativo y expansión progresiva de la nasalidad}

Como se dijo anteriormente, el prefijo causativo exhibe una forma oral $m b \dot{t}$ - cuando se combina a una raíz léxica oral (83) y una forma nasal $m \dot{t}$ - cuando se combina con una raíz léxica nasal(84). En otras palabras, su forma (i.e. oral o nasal) está sujeta a la expansión regresiva de la nasalidad desencadenada por la calidad nasal de la raíz léxica.

(83) mbì-rìri

CAUS1-temblar

hace que tiemble

(84) mí-mbowa

CAUS1-tamiz

hace que tamice

Sin embargo, hay ejemplos en los que el prefijo 'CAUS1' no experimenta cambios debido a la expansión regresiva de la nasalidad. En esos casos, su variante nasal $m \dot{t}$ - afecta la calidad oral de las consonantes sordas siguientes a la raíz léxica, las que se convierten en consonantes sonoras prenasalizadas, producidas en el mismo punto de articulación de su contraparte oral. Este proceso afecta principalmente las raíces léxicas cuyo primer segmento es una oclusiva velar sorda $[\mathrm{k}]$, que se transforma en una oclusiva sonora prenasalizada [ng]. Los ejemplos (85) - (91) muestran la alternancia [k] [ng]. 
a.

a-kiye
1SGAC-miedo
tengo miedo

(86) a

a.

a-kwaru
1SGAC-orinar
orino

(87)
a.
kìri
cosquilloso
(es) cosquilloso

(88) a. a-karu

1SGAC-comer

como/comí

(89) a. a-kwera

1SGAC-sanar

me sano

(90) a. ki’a
sucio
(es) sucio

(91) a. ku'i

molido b. mi-ngiye

CAUS1-asustar

(lo) asusta

b. mi-ngwaru

CAUS1-orinar

(lo) hace orinar

b. ha'e mi-ngini

él/ella CAUS1-cosquilloso

él / ella le hace cosquillas

b. mi-ngaru

CAUS1-comer

(lo) alimenta

b. a-mi-ngwére-ma

1SGAC-CAUS1-curar-RES

(lo) tranquilizo

b. mi-ngi'a

CAUS1-sucio

(lo) ensucia

b. pu-mu-ngu'i-po

2PLAC-CAUS1-aflojar-FUT

(ustedes) aflojarán (eso)

Hay dos raíces léxicas cuya primera consonante africada palatal sorda $\left.\left[\mathrm{t} \int\right]<\mathrm{ch}\right\rangle \mathrm{se}$ transforma en una africada palatal sonora pre-nasalizada [dn3] <ny > (92).

\section{$(92)$}
a.
a-che
1SGAC-dormir
duermo
b. ai-che
1SGAC-enter
entro
b. mo-nye
US1-dormi
(lo) hago dormir
b. mi-nye
CAUS1-enter

(lo) puso dentro (lit. hizo que entrara)

Finalmente, hay una raíz léxica cuya primera consonante fricativa alveolar sorda [s] se transforma en una oclusiva alveolar sonora prenasalizada [nd] (93).
(93) a.
soso
perforar
perfora
b. nde mí-ndoso
vos CAUS1-cortar
cortás


Como se vio en la Sección 6.3, las consonantes prenasalizadas [mb] y [nd] pueden ocurrir en posición inicial y media, mientras que la consonante velar prenasalizada [ng] ocurre sólo en posición media y, en muchas lenguas de la familia, en el límite de morfemas (Costa 2007, Grenand 1980:34). Estos dos grupos de segmentos, se distinguen, además, por los procesos en los que intervienen: [ $\mathrm{\eta g}$ ] resulta de procesos morfo-fonológicos en virtud de los cuales una raíz nasal prenasaliza la primera consonante del sufijo ([k] $\rightarrow$ [ng] $)$ o un prefijo nasal prenasaliza la primera consonante de una raíz verbal oral. Este es el caso específico del prefijo causativo $m \dot{t}$ - en tapiete, como se vio en los ejemplos (85)- (91).

La expansión progresiva de la nasalidad desencadenada por la variante nasal del prefijo causativo $m \dot{t}$ - está, no obstante, limitada a las raíces verbales dadas en los ejemplos precedentes. Por lo tanto, la regla que determina la expansión de la nasalidad en este contexto no es productiva, ya que se aplica solamente a un conjunto de raíces verbales ${ }^{23}$. Los ejemplos que se presentan en (94) muestran cómo la primera consonante de las raíces verbales preserva su calidad oral a pesar de estar precedida por la forma nasal del prefijo causativo.

(94) a. a-min-kambu

1SGAC-CAUS1-mamar

le hago mamar

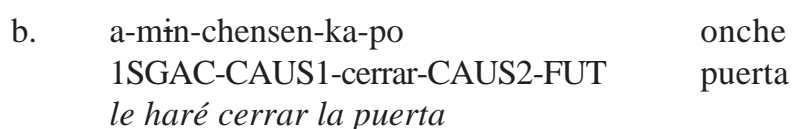

\subsection{Acento}

Como se señaló en la sección 5, el acento es predecible, ya que siempre recae sobre la penúltima silaba. Este patrón experimenta algunas modificaciones como consecuencia de la concatenación de sufijos y clíticos que se discuten a continuación.

\subsubsection{Sufijos y clíticos y el lugar del acento}

El lugar del acento dentro de una palabra fonológica puede modificarse según se apliquen las reglas de acentuación antes o después de la concatenación de morfemas a la raíz léxica. Cuando las reglas de acentuación se aplican antes de que los afijos se combinen con la raíz léxica, la forma resultante se acomoda al patrón por defecto del acento (es decir, la penúltima sílaba). Los sufijos involucrados en este proceso se enumeran en la 6.

${ }^{23}$ Dietrich (1986:104) reporta un proceso similar para el ava-guaraní (chiriguano), de acuerdo al cual tres raíces verbales orales (óki 'llueve', akìye 'tengo miedo' y ápa 'me despierto') al combinarse con el prefijo causativo mbo- mo- sufren la modificación de la oclusiva velar [k] que se convierte en

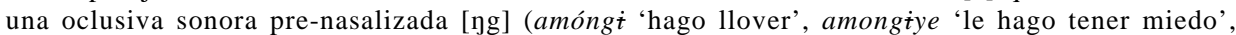
amómba 'despierto a alguien' ). Igualmente, Costa (2003:50-51) y Rose (2003:653) observan el mismo fenómeno en nhandeva y emerillón, respectivamente. 


\begin{tabular}{|l|l|l|l|l|}
\hline Sufijo & Glosa & Ejemplo & & Traducción \\
\hline \hline -ä & NEG & a-karu-ä & [a-karúã] & 'no comí' \\
\hline -ka & CAUS2 & a-henon-ka & [ahenónka] & 'lo hago llamar' \\
\hline -ha & 1PLEXCL & ndi-reka-ha & [ndirekáha & '(alguien) te busca' \\
\hline -kwe & AUM & ro'ł-kwe & [rớkwe] & 'helado grande' \\
\hline -ite & SUP & potá-ite & [potájte] & 'quiere mucho' \\
\hline -se & DES & ma-mbe'u-se & [mambe?úse] & 'chismoso' \\
\hline
\end{tabular}

Tabla 6. Sufijos monosilábicos que atraen el acento

Cuando las reglas de acentuación se aplican después de que los morfemas se combinan con la raíz léxica, el acento recae sobre la antepenúltima sílaba de la palabra resultante. Los morfemas involucrados en este proceso pueden considerarse clíticos y se enumeran en la Tabla 7.

\begin{tabular}{l|l|ll|l}
\hline Sufijo & Glosa & Ejemplo & & Traducción \\
\hline \hline -wa / -a & NOM & páye-a & [pázea] & 'curandero' \\
\hline -e & REC & ó-ho-e & [hóhoe] & 'el/la acaba de irse' \\
\hline -pe & LOC & ténta-pe & [téntape] & 'en el pueblo' \\
\hline -ipi & LOCMOV & ténta-ipi & [téntajpi] & 'a través del pueblo' \\
\hline -ma & RES & a-mi-ngwére-ma & [amingwérama] & '(lo) tranquilicé' \\
\hline -pi & INST & shi-lapi a-mbapára-pi [jilápi ambapárapi] & 'escribo con un lápiz' \\
\hline -po & FUT & a-máma-po & [amámapo] & 'lo conseguiré' \\
\hline -kwi & FUTDIST & a-káru-kwi & [akárukwi] & 'comeré' \\
\hline -kwe & PAS & a-púka-kwe & [apúkakwe] & 'me reí' \\
\hline -yi & FRC & a-ñóno- yi & [anónozi] & '(lo) pongo otra vez' \\
\hline -re & DIR & kúpe-re mbipara & [kúpere mbipára] & 'dibujá en la espalda' \\
\hline -mba & NEG & a-káru-mba a-iko & [akárumba ájko] & 'estoy sin comer' \\
\hline -nda & EVD & há'e-nda & [háPenda] & 'se dice que él...' \\
\hline
\end{tabular}

Tabla 7. Sufijos monosilábicos que no atraen el acento

En suma, sólo la penúltima y la antepenúltima sílabas pueden acentuarse en tapiete. De esta manera, mientras que la penúltima sílaba constituye la ubicación por defecto del acento en palabras no derivadas, el acento en la antepenúltima sílaba es el resultado de la aplicación de reglas de acentuación una vez que los morfemas se han combinado con la raíz léxica.

\section{CONCLUSIÓN}

En este artículo se han examinado las principales características de la fonología segmental del tapiete. El artículo ha esbozado las reglas que gobiernan las variaciones 
alofónicas y ha abordado la fonotáctica de la lengua a través de un examen de las tendencias que subyacen a la co-ocurrencia de los segmentos dentro de una palabra de tipo CV.CV. La estructura silábica canónica del tapiete es de tipo $(\mathrm{C}) \mathrm{V}(\mathrm{V})(\mathrm{N})$, donde todas las consonantes pueden ocurrir en posición inicial de sílaba y, a excepción de la oclusiva glotal [?] y de la oclusiva sonora velar prenasalizada [ng], todas pueden ocurrir en posición inicial de palabra. La nasalidad en tapiete es un rasgo suprasegmental que se expande regresiva y progresivamente a partir de una sílaba nasal acentuada y cuyo dominio es la palabra fonológica. En este contexto, las oclusivas sonoras prenasalizadas, aunque fonéticamente complejas, fonológicamente son consideradas segmentos simples que ocupan la posición de ataque de la sílaba. Por su parte, las oclusivas sordas prenasalizadas resultan de alternancias fonológicas producto de la armonía nasal; por lo tanto, son consideradas como una secuencia de segmentos cuya porción nasal ocupa la posición de coda de la sílaba precedente, y la porción oclusiva ocupa la posición de ataque de la sílaba siguiente. Las consonantes nasales en posición de coda se encuentran solamente en el contexto de la armonía nasal y, en consecuencia, son fonológicamente irrelevantes en esta posición. Finalmente, el acento es predecible, ya que siempre recae sobre la penúltima silaba aunque este patrón experimenta algunas modificaciones como consecuencia de la concatenación de sufijos y clíticos.

\section{BIBLIOGRAFÍA}

BARRATT, Leslie. (1981) Prenasalized stops in Guaraní. Where the autosegment fails. Linguistic Analysis 7:187-202.

CANESE, Natalia. (1994) Gramática Guaraní. Asunción: Colección Nemity.

COSTA, Consuelo de Paiva Godinho. (2003) Nhandewa aywu. Dissertação de Mestrado em Lingüística. Campinas: Instituto de Estudos da Linguagem, Universidade Estadual de Campinas.

.(2007) Apyngwa rupigwa: nasalização em Nhandewa-Guaraní. Tese de Doutorado em Lingüística. Campinas: Instituto de Estudos da Linguagem, Universidade Estadual de Campinas.

DIETRICH, Wolf. (1986) El Idioma Chiriguano: Gramática, textos, vocabulario. Madrid: Instituto de Cooperación Iberoamericana.

(1990) More evidence for an internal classification of tupi-guarani languages. Indiana, Suplement 12. Berlin: Gebr. Mann.

DOOLEY, Robert A. (1984) Nasalização na língua Guaraní. En Robert A. Dooley (org.). Estudos sobre línguas tupí do Brasil, pp. 7-35. (Série Lingüística 11). Brasília: SIL.

FARGETTI, Cristina Martins. (2001) Estudo fonológico e morfossintático da língua Juruna. Tese de Doutorado em Lingüística. Campinas: Instituto de Estudos da Linguagem, Universidade Estadual de Campinas.

GOLDSMITH, John A. (1999) Phonological Theory. The Essential Readings. Oxford: Blackwell Publishers.

GOMES, Dioney Moreira. (2006) Estudo morfológico e sintático da língua Mundurukú (Tupí). Tese de Doutorado em Lingüística. Brasília: Departamento de Lingüística, Línguas Clássicas e Vernácula, Universidade de Brasília. 
GONZÁLEZ, Hebe. (2005) A grammar of Tapiete (Tupi-Guarani). Ph.D Thesis. Pittsburgh: Department of Linguistics, University of Pittsburgh.

GREGORES, Emma; SUÁREZ, Jorge A. (1967) A description of colloquial guaraní. The Hague - Paris: Mouton \& Co.

GRENAND, Françoise. (1980) La langue Wayãpi (Guyane française): Phonologie et grammaire. Paris: Centre National de la Recherche Scientifique.

GUEDES, Marymarcia. (1991) Subsídios para uma análise fonológica do Mbyá. Dissertação de Mestrado em Lingüística. Campinas: Instituto de Estudos da Linguagem, Universidade Estadual de Campinas.

HARRISON, Carl H. ; TAYLOR, John M. (1971) Nasalization in kaiwá. En David Bendor-Samuel (ed.). Tupi Studies I, pp. 15-20. Norman, Oklahoma: SIL.

JENSEN, Cheryl. (1990) Cross-Referencing changes in some Tupí-Guaraní languages. En Doris. L. Payne (ed.). Amazonian Linguistics: Studies in Lowland South American Languages, pp. 117158. Austin: University of Texas Press.

JENSEN, Cheryl. (1998) Comparative Tupí-Guaraní morphosyntax. En D. C. Derbyshire y G. K. Pullum (eds.). Handbook of Amazonian Languages, vol. 4: 489-618. Berlin/New York: Mouton de Gruyter.

KENSTOWICZ, Michael. (1994) Phonology in Generative Grammar. Cambridge, Mass.:Blackwell Publishers.

MAGALHÃES, Marina Maria Silva. (2007) Sobre a morfologia e a sintaxe da língua Guajá (Família Tupí-Guaraní). Tese de Doutorado em Lingüística. Brasília: Departamento de Lingüística, Português e Línguas Clássicas, Universidade de Brasília.

McCARTHY, John J.; PRINCE, Alan S. (1999) Prosodic Morphology (1986). En John A. Goldsmith (ed.). Phonological Theory. The Essential Readings, pp. 238-288. Oxford: Blackwell Publishers.

NASCIMENTO, Ana Paula Lion Mamede. (2008) Estudo fonético y fonológico da língua Guajá. Tese de Mestrado em Lingüística. Brasília: Departamento de Lingüística, Português y Línguas Clássicas, Universidade de Brasília

PICANÇO, Gessiane Lobato. (2005) Mundurukú: phonetics, phonology, synchrony, diachrony. Ph.D Thesis. Columbia: University of British Columbia

PRAÇA, Walkíria Neiva. (2007) Morfossintaxe da língua Tapirapé (Família Tupí-Guaraní). Tese de Doutorado em Lingüística. Brasília: Departamento de Lingüística, Português Línguas Clássicas, Universidade de Brasília.

PRIEST, Perry N. (1980) Estudios sobre el idioma siriono. vol. 10. Riberalta: ILV/Ministerio de Educación y Cultura.

RODRIGUES, Aryon D. (1984/1985) Relações internas na família lingüística Tupi-Guarani. Revista de Antropologia 27/28: 3-53.

ROSE, Françoise. (2001-2002) Le problème de la nasalité dans l'inventaire phonologique de l'émerillon. Amerindia 26/27:149-172.

.(2003). Morphosyntaxe de l'Emérillon (Langue Tupi-Guarani de Guyane française). Thèse de Doctorat. Lyon: Département de Sciences du Langage et Arts - Faculté des Lettres, Sciences du Langage et Arts, Université Lumière Lyon 2. 
GoNZÁLEZ: UNA APROXIMACIÓN A LA FONOLOGÍA DEL TAPIETE (TuPí-GUARANí)

SAELZER, M. (1976) Fonologia provisória da língua Kamayurá. Brasília: SIL.

SILVA, Márcio Ferreira da (1981) A fonologia segmental Kamayurá. Dissertação de Mestrado em Lingüística. Campinas: Instituto de Estudos da Linguagem, Universidade Estadual de Campinas.

Recebido: 30/4/2008

Versão revista: $15 / 12 / 2008$

Aceito: 6/1/2009 